\title{
OPERATORS ON TENSOR PRODUCTS OF BANACH SPACES
}

BY

\section{TAKASHI ICHINOSE}

ABSTRACT. The present paper is a study of operators on tensor products of Banach spaces with the notion of maximal extensions introduced by G. Köthe such that the closure of a closable operator is its unique maximal extension. For a class of such operators the spectral mapping theorem is established. The results apply to the operator $A \otimes I+I \otimes B$ and give a new meaning to the method of separation of variables.

Introduction. Given densely defined linear operators $A$ and $B$ in Banach spaces $X, Y$, respectively, we assign to each polynomial in $\xi$ and $\eta$

$$
P(\xi, \eta)=\sum c_{j k} \xi^{j} \cdot \eta^{k}
$$

two kinds of polynomial operators

$$
\sum c_{j k} A^{j} \otimes B^{k}=P(A \otimes I, I \otimes B)
$$

and

$$
\sum c_{j k} A^{j} \underset{\alpha}{\hat{\otimes}} B^{k}
$$

defined in the tensor product $X \hat{\otimes}_{\alpha} Y$, the completion of $X \otimes Y$ with respect to a reasonable norm $a \cdot A^{j} \hat{\otimes}_{a} B^{k}$ denotes a maximal extension of $A^{j} \otimes B^{k}$ in $X \hat{\otimes}_{\alpha} Y$. If $A$ and $B$ are bounded, it has been shown by M. Schechter [19] and the author [10] that the following spectral mapping theorem holds independently of a given uniform reasonable norm $a: P(\sigma(A), \sigma(B))=\sigma(P(A \otimes I, I \otimes B))$. This is a classical result of $C$. Stéphanos when $X$ and $Y$ are finite dimensional so that $A$ and $B$ may be represented as matrices (see [15]). A further extension of this result is obtained in [10] for not necessarily bounded operators and polynomials continuous at the extended spectra. But the class of polynomials there is rather restricted.

The main aim of the present paper is to establish the spectral mapping theorem for a larger class of polynomials, though imposing certain restrictions on the operators. In particular, our theory applies to the operator $A \otimes I+I \otimes B$ and extends the results of H. O. Cordes [4], Ju. M. Berezanskii [2], L. and K. Maurin

Received by the editors May 25, 1971.

AMS 1970 subject classifications. Primary 47A10, 47A60.

Key words and phrases. Topological tensor products, operators on tensor products, spectral mapping theorem, spectrum, separation of variables. 
[16] for selfadjoint operators. The spectral mapping theorem imparts thereby a new meaning to the method of separation of variables (cf. B. Friedman [5]).

$\S_{1}$ is concerned with polynomial operators and the closability of the operators of both (1.2) and (1.3). In $\$ 2$ the spectral mapping theorem for the operators of the kind (1.2) is presented. In $\$ 3$ we show for a faithful uniform reasonable norm that the closures of both the operators (1.2) and (1.3) coincide. This fact reduces the spectral mapping theorem for the operators of the kind (1.2) to that for the operators of the kind (1.3) since the spectrum is unchanged under the closure operation. $\$ 4$ is devoted as a special case to polynomial operators of the first order, which were treated in [2] and [16]. We consider fractional powers and present as a special case a generalization of the results by V. P. Mihaulov [17] for the first boundary value problem of quasi-elliptic differential equations.

Finally we note that assumption of the faithfulness of the norm $\alpha$ will facilitate all the considerations.

For the basic notions and results on linear operators and tensor products used here, we follow [14], [7], [9] and [10] (cf. [8], [13], [20], [6], [18]).

1. Polynomial operators. Throughout, $X$ and $Y$ will denote complex Banach spaces and $a$ a reasonable norm on the tensor product $X \otimes Y$, i。e. a crossnorm whose dual norm $a^{\prime}$ is also a crossnorm on $X^{\prime} \otimes Y^{\prime} . X \hat{\otimes}_{\alpha} Y$ is the completion of $X \otimes Y$ with respect to $a$.

Let $A: D[A] \subset X \rightarrow X ; B: D[B] \subset Y \rightarrow Y$ be linear operators with spectra $\sigma(A), \sigma(B)$ and with resolvent sets $\rho(A), \rho(B)$.

To each polynomial of degrees $m$ in $\xi$ and $n$ in $\eta$

$$
P(\xi, \eta)=\sum c_{j k} \xi^{j} \cdot \eta^{k} \quad\left(c_{j k}: \text { complex }\right)
$$

we assign the following two kinds of polynomial operators in $X \hat{\otimes}_{\alpha} Y$

$$
\sum c_{j k} A^{j} \otimes B^{k}=P(A \otimes I, I \otimes B)
$$

with domain

$$
\begin{aligned}
D[P(A \otimes I, I \otimes B)] & \equiv \bigcap_{j, k ; c ; k \neq 0} D\left[A^{j} \otimes B^{k}\right] \\
& \equiv \bigcap_{j, k ; c_{j k} \neq 0} D\left[A^{j}\right] \otimes D\left[B^{k}\right]=D\left[A^{m} \otimes B^{n}\right] \\
& \equiv D\left[A^{m}\right] \otimes D\left[B^{n}\right]
\end{aligned}
$$

(see [10] or use [9, Lemma 4.20]), and

$$
\sum c_{j k} A^{j} \underset{a}{\hat{\otimes}} B^{k}
$$


with domain $D\left[\sum c_{j k} A^{j} \hat{\otimes}_{a} B^{k}\right] \equiv \bigcap_{j, k ; c j k \neq 0} D\left[A^{j} \hat{\otimes}_{a} B^{k}\right]$. Here $A^{j} \hat{\otimes}_{a} B^{k}$ denotes a maximal extension of $A^{j} \otimes B^{k}$ in $X \hat{\otimes}_{\alpha} Y$ for each pair $(j, k)$ of nonnegative integers. $I$ will denote the identity operator in both $X$ and $Y$, and $I \hat{\otimes}_{\alpha} I$ in $X \hat{\otimes}_{\alpha} Y$. The polynomial operators (1.2) and (1.3) have maximal extensions in $X \hat{\otimes}_{\alpha} Y$, which we shall denote by $\widetilde{P}(A \otimes I, I \otimes B)$ (cf. $\Theta(P ; A, B)$ in the notation of $[10])$ and $\left(\sum c_{j k} A^{j} \hat{\otimes}_{a} B^{k}\right)^{\sim}$, respectively. When they are closable, their closures are the ir unique maximal extensions. So we shall employ the same notations for the closures (cf. [14]).

A reasonable norm $a$ is said to be faithful if the natural continuous linear mapping of $X \hat{\otimes}_{\alpha} Y$ into $X \hat{\otimes}_{\epsilon} Y$ is one-to-one. If $X$ and $Y$ contain Schauder bases, every uniform reasonable norm on $X \otimes Y$ is faithful (see [6]).

Theorem 1.1. If $A$ and $B$ are closable and have nonempty resolvent sets $\rho(A), \rho(B)$, then the polynomial operators (1.2) and (1.3) are closable in $X \hat{\otimes}_{a} Y$, provided $\alpha$ is a faithful reasonable norm on $X \otimes Y$ (cf. [9], [10]).

Proof. It suffices to show the closability of (1.3), since (1.3) is an extension of (1.2). We may assume $A$ and $B$ closed. For simplicity further assume them densely defined. Then, since $\rho(A)$ and $\rho(B)$ are nonempty, $A^{j}$ and $B^{k}$ are also densely defined and closed (e.g. [8]). Since

$$
D\left[A^{m}\right] \otimes D\left[B^{n}\right] \subset D\left[\sum c_{j k} A^{j} \underset{\alpha}{\hat{\otimes}} B^{k}\right] \equiv \bigcap_{j, k ; c} D\left[A_{j k}^{j} \underset{\alpha}{\hat{\otimes}} B^{k}\right],
$$

the operator (1.3) is densely defined in $X \hat{\otimes}_{\alpha} Y$, so that its adjoint is well defined. We have in general

$$
\begin{aligned}
\left(\sum c_{j k} A^{j} \underset{a}{\hat{\otimes}} B^{k}\right)^{\prime} & \supset \sum c_{j k}\left(A^{j} \underset{a}{\hat{\otimes}} B^{k}\right)^{\prime}=\sum c_{j k}\left(A^{j} \otimes B^{k}\right)^{\prime} \\
& \supset \sum c_{j k}\left(A^{\prime}\right)^{j} \otimes\left(B^{\prime}\right)^{k}=P\left(A^{\prime} \otimes I^{\prime}, I^{\prime} \otimes B^{\prime}\right),
\end{aligned}
$$

for a densely defined linear operator and its maximal extension have the same adjoint. If $A$ is closed with $\rho(A) \neq \varnothing, D\left[\left(A^{\prime}\right)^{j}\right]$ is dense in $X_{s}^{\prime}$, where $X_{s}^{\prime}$ denotes the dual space $X^{\prime}$ equipped with the weak topology defined by the dual pair $\left\langle X^{\prime}, X\right\rangle$. The same is true for $B$.

Since $\alpha$ is faithful and since every element of $X \hat{\otimes}_{\epsilon} Y$ is separately continuous bilinear on $X_{s}^{\prime} \times Y_{s^{\prime}}^{\prime}, D\left[P\left(A^{\prime} \otimes I^{\prime}, I^{\prime} \otimes B^{\prime}\right)\right]=D\left[\left(A^{\prime}\right)^{m}\right] \otimes D\left[\left(B^{\prime}\right)^{n}\right]$ is dense in $\left(X \hat{\otimes}_{a} Y\right)^{\prime}$ with respect to the weak topology defined by the dual pair $\left\langle\left(X \hat{\otimes}_{a} Y\right)^{\prime}, X \hat{\otimes}_{a} Y\right\rangle$, so that the domain of $\left(\sum c_{j k} A^{j} \hat{\otimes}_{a} B^{k}\right)^{\prime}$ is dense in $\left(X \hat{\otimes}_{a} Y\right)^{\prime}$ in the same weak topology. This proves the closability of (1.3). Q.E.D.

If $E$ is a Banach space and $T: D[T] \subset E \rightarrow E$ is a linear operator with domain $D[T]$ and range $R[T]$ in $E, G(T)$ denotes the graph of $T$. Every maximal extension $\widetilde{T}$ of $T$ has the same domain $D[\widetilde{T}]=H_{T}$ which is the projection of the 
closure $\overline{G(T)} \subset E \times E$ into the first $E$. The singularity $S[T]$ of $T$ is by definition the set of all $y \in E$ such that $(0, y) \in \overline{G(T)}$. $S[T]$ is a closed subspace of $E$ and $S[\tilde{T}]=S[T] . T$ is closable if and only if $S[T]=0$ (see [14]).

Proposition 1.2. Let a be a reasonable norm on $X \otimes Y$. Let $A$ and $B$ be densely defined maximal linear operators. Unless one of the extended spectra $\sigma_{e}(A)$ and $\sigma_{e}(B)$ contains 0 while the other contains $\infty$, then

(1.5) $D[A \underset{\alpha}{\otimes} B] \cap(X \otimes Y) \equiv H_{A \otimes B} \cap(X \otimes Y)=D[A] \otimes D[B] \equiv D\left[\begin{array}{l}A \otimes B \\ \otimes\end{array}\right.$

Remark 1.3. The assumption for $A$ and $B$ in Proposition 1.2 is equivalent to the following condition consisting of three mutually exclusive parts ([9], [10]):

(i) $A$ and $B$ are bounded;

(ii) $A$ and $B$ have densely defined bounded inverses;

(iii) one of $A$ and $B$ is bounded and has a densely defined bounded inverse while the other is arbitrary.

Proof of Proposition 1.2. The inclusion $D[A] \otimes D[B] \subset D\left[A \hat{\otimes}_{\alpha} B\right] \cap(X \otimes Y)$ is obviously true.

To prove the reverse inclusion let $u \in D\left[A \hat{\otimes}_{\alpha} B\right] \cap(X \otimes Y)$. We may assume $u \neq 0 . u$ has an expression $\sum_{j=1}^{r} x_{j} \otimes y_{j}$ in which both the sequences $\left\{x_{j}\right\}_{j=1}^{r}$ and $\left\{y_{j}\right\}_{j=1}^{r}$ are linearly independent and the number $r$, i.e. the rank of $u$, is determined uniquely. Then, for $\left(x^{\prime}, y^{\prime}\right) \in D\left[A^{\prime}\right] \times D\left[B^{\prime}\right]$,

$$
\left\langle A \underset{a}{\hat{\otimes}} B u, x^{\prime} \otimes y^{\prime}\right\rangle=\left\langle u,(A \underset{a}{\hat{\otimes}} B)^{\prime}\left(x^{\prime} \otimes y^{\prime}\right)\right\rangle=\left\langle u, A^{\prime} x^{\prime} \otimes B^{\prime} y^{\prime}\right\rangle
$$

$$
=\sum_{j=1}^{r}\left\langle x_{j}, A^{\prime} x^{\prime}\right\rangle\left\langle y_{j}, B^{\prime} y^{\prime}\right\rangle .
$$

The proof will be divided into three cases corresponding to the equivalent condition reformulated in Remark 1.3.

(i) (1.5) is obviously true, since $D[A]=X$ and $D[B]=Y$ by maximality.

(ii) We have $0 \in \rho(A)=\rho\left(A^{\prime}\right)$ and $0 \in \rho(B)=\rho\left(B^{\prime}\right)$.

Since the adjoint operators are always closed, $A^{\prime}$ maps $D\left[A^{\prime}\right]$ onto $X^{\prime}$. Since the $x_{j}$ are linearly independent, there exists a sequence $\left\{x_{j}^{\prime}\right\}_{j=1}^{r}$ in $D\left[A^{\prime}\right]$ with $\left\langle x_{j}, A^{\prime} x_{k}^{\prime}\right\rangle=\delta_{j k}$ for $j, k=1,2, \cdots, r$. It follows from (1.6) that, for all $y^{\prime} \epsilon$ $D\left[B^{\prime}\right]$,

$$
\left\langle A \underset{a}{\otimes} B u, x_{k}^{\prime} \otimes y^{\prime}\right\rangle=\left\langle y_{k^{\prime}}, B^{\prime} y^{\prime}\right\rangle, \quad k=1,2, \cdots, r
$$

so that, since the dual norm $\alpha^{\prime}$ is also reasonable and $B$ is maximal, we have $y_{k} \in D[B]$ for $k=1,2, \cdots r$. 
Choosing a sequence $\left\{y_{j}^{\prime}\right\}_{j=1}^{r}$ in $D\left[B^{\prime}\right]$ with $\left\langle y_{j}, B^{\prime} y_{k}^{\prime}\right\rangle=\delta_{j k}$, we conclude similarly $x_{k} \in D[A]$ for $k=1,2, \cdots, r$.

(iii) Without loss of generality we may assume $A$ is bounded with a densely defined bounded inverse. Then by maximality $D[A]=X$, so $D\left[A^{\prime}\right]=X^{\prime}$. Since $0 \in \rho(A)=\rho\left(A^{\prime}\right)$, the proof in (ii) yields $y_{k} \in D[B]$ for $k=1,2, \cdots, r$. Q.E.D.

Recall that if $T$ is closed or if $T$ is one-to-one and open, then $R[T] \cap$ $S[T]=0$ (cf. [14]), in order to understand better the condition of the following

Proposition 1.4. Let a be a reasonable norm on $X \otimes Y$. Let $A$ and $B$ be densely defined maximal linear operators. If $A$ is one-to-one and $R[A] \cap S[A]$ $=0$, then

$$
\begin{aligned}
D[A \underset{\alpha}{\hat{\otimes}} B] \cap(D[A] \otimes Y) & \equiv H_{A \otimes B} \cap(D[A] \otimes Y) \\
& =D[A] \otimes D[B] \equiv D[A \otimes B] .
\end{aligned}
$$

Proof. Let $u=\sum_{j=1}^{r} x_{j} \otimes y_{j} \in D\left[A \hat{\otimes}_{a} B\right] \cap(D[A] \otimes Y), u \neq 0$, where both the sequences $\left\{x_{j}\right\}_{j=1}^{r} \subset D[A]$ and $\left\{y_{j}\right\}_{j=1}^{r} \subset Y$ are linearly independent. Then it follows from (1.6) that

$$
\left\langle A \underset{\alpha}{\hat{\otimes}} B u, x^{\prime} \otimes y^{\prime}\right\rangle=\sum_{j=1}^{r}\left\langle A x_{j}, x^{\prime}\right\rangle\left\langle y_{j}, B^{\prime} y^{\prime}\right\rangle .
$$

Since $A$ is one-to-one, the $A x_{j}$ as well as the $x_{j}$ are linearly independent. Since $\left\langle X / S[A], D\left[A^{\prime}\right]\right\rangle$ is a dual pair and $R[A] \cap S[A]=0$, there exists a sequence $\left\{x_{j}^{\prime}\right\}_{j=1}^{r}$ in $D\left[A^{\prime}\right]$ with $\left\langle A x_{j}, x_{k}^{\prime}\right\rangle=\delta_{j k}$ for $j, k=1,2, \cdots, r$. A similar argument to the proof of Proposition 1.2 concludes $y_{k} \in D[B]$ for $k=1$, $2, \cdots, r$. Q.E.D.

Remark 1.5. The following counterexamples show Propositions 1.2 and 1.4 fail if the assumptions there are not satisfied.

(1) Recall Counterexample 4.18 in [9]. Let $X=Y=l^{2}$ and let $\left\{e_{\nu}\right\}_{\nu=1}^{\infty}$ be the canonical orthogonal basis in $l^{2}$. For a certain uniform reasonable norm $\alpha_{0}$, $l^{2} \hat{\otimes}_{a_{0}} l^{2}$ is identified with the Hilbert space $\left\{\left(c_{m n}\right) ; \sum_{m, n=1}^{\infty}\left|c_{m n}\right|^{2}<\infty\right\}$. Define $A$ with $D[A]=l^{2}$ by

$$
A e_{m}=0 \text { for } m=1, m>2 ; \quad A e_{2}=e_{1},
$$

and $B$ with $D[B]=\left\{\left(c_{n}\right) \in l^{2} ; \sum_{n=1}^{\infty}\left|n c_{n}\right|^{2}<\infty\right\}$ by

$$
B e_{n}=n e_{n} \text { for } n=1,2, \cdots \text {. }
$$

$A$ is bounded and $B$ is closed. $\sigma(A)=\{0\}$ and $\sigma(B)=\{n\}_{n=1}^{\infty}$. The norm $\alpha_{0}$ is necessarily faithful, so $A \hat{\otimes}_{a_{0}} B$ is closed in $l^{2} \hat{\otimes}_{a_{0}} l^{2} . D\left[A \hat{\otimes}_{a_{0}} B\right]=$ $\left\{\left(c_{m n}\right) \in l^{2} \hat{\otimes}_{a_{0}} l^{2} ; \sum_{n=1}^{\infty}\left|n c_{2 n}\right|^{2}<\infty\right\}$. Let $b=(1,1 / 2,1 / 3, \cdots) \in l^{2}$. Then 
$b-(1 / 2) e_{1} \in D[A]=l^{2}$ and $b \notin D[B]$. But $\left(b-(1 / 2) e_{1}\right) \otimes b \in D\left[A \hat{\otimes}_{a_{0}} B\right]$, since all the coefficients $c_{2 n}$ of $\left(b-(1 / 2) e_{1}\right) \otimes b=\sum_{m, n} c_{m n}\left(e_{m} \otimes e_{n}\right)^{0}$ vanish.

(2) We consider in the same Hilbert space $l^{2} \hat{\otimes}_{a_{0}} l^{2}$ as in (1). Let $B$ be the same operator in $l^{2}$ as in (1). Define $A$ with $D[A]=\left\{\left(c_{m}\right) \in l^{2} ; \sum_{m=3}^{\infty}\left|m c_{m}\right|^{2}<\infty\right\}$ by $A e_{1}=A e_{2}=0$ and $A e_{m}=m e_{m}$ for $m>2$.

$A$ is closed but not one-to-one. $A \hat{\otimes}_{a_{0}} B$ is closed in $l^{2} \hat{\otimes}_{a_{0}} l^{2}$ with domain $D\left[A \hat{\otimes}_{a_{0}} B\right]=\left\{\left(c_{m n}\right) \in l^{2} \hat{\otimes}_{a_{0}} l^{2} ; \sum_{m=3}^{\infty} \sum_{n=1}^{\infty}\left|m n c_{m n}\right|^{2}<\infty\right\}$. Let $a=\left(1,1 / 2^{2}\right.$, $\left.1 / 3^{2}, \cdots\right) \in l^{2}$ and $b=(1,1 / 2,1 / 3, \cdots) \in l^{2}$. Then $a+e_{1}$ and $a+e_{2}$ are in $D[A]$ and linearly independent. $b$ and $a+b$ are linearly independent and do not lie in $D[B]$. But $\left(a+e_{1}\right) \otimes(a+b)+\left(a+e_{2}\right) \otimes(-b)$ lies in $D\left[A \hat{\otimes}_{a_{0}} B\right]$.

In what follows, we study how $A^{j} \hat{\otimes}_{\alpha} B^{k}$ is related to $A \hat{\otimes}_{\alpha} I$ and $I \hat{\otimes}_{\alpha} B$.

Proposition 1.6. Besides the condition of Proposition 1.2 assume $a$ is uniform, and $A$ and $B$ are closed. Then for any maximal extensions $A \hat{\otimes}_{\alpha} I$ of $A \otimes I$ and $I \hat{\otimes}_{a} B$ of $I \otimes B$, the closures of the graphs of $\left(A \hat{\otimes}_{a} I\right)\left(I \hat{\otimes}_{\alpha} B\right)$, $\left(I \hat{\otimes}_{a} B\right)\left(A \hat{\otimes}_{a} I\right)$ and $A \otimes B$ coincide. Any maximal extension $A \hat{\otimes}_{a} B$ of $A \otimes B$ equals $\left(A \hat{\otimes}_{a} I\right)\left(I \hat{\otimes}_{\alpha} B\right)$ and $\left(I \hat{\otimes}_{a} B\right)\left(A \hat{\otimes}_{a} I\right)$ for some maximal extensions $A \hat{\otimes}_{a} I$ and $I \hat{\otimes}_{a} B$.

If $\alpha$ is, in addition, faithful then

$$
A \underset{a}{\hat{\otimes}} B=(A \underset{a}{\hat{\otimes}} I)(I \underset{a}{\ddot{\otimes}} B)=(I \underset{a}{\hat{\otimes}} B)(A \underset{a}{\hat{\otimes}} I) .
$$

Proof. The proof will be divided into three cases corresponding to the equivalent condition reformulated in Remark 1.3 (cf. proof of Proposition 1.2).

(i) As $\alpha$ is uniform, $A \hat{\otimes}_{\alpha} B, A \hat{\otimes}_{\alpha} I$ and $I \hat{\otimes}_{\alpha} B$ are nothing but the continuous extensions of $A \otimes B, A \otimes I$ and $I \otimes B$, respectively, to the entire space $X \hat{\otimes}_{\alpha} Y$, so that $A \hat{\otimes}_{a} B=\left(A \hat{\otimes}_{\alpha} I\right)\left(I \hat{\otimes}_{\alpha} B\right)=\left(I \hat{\otimes}_{a} B\right)\left(A \hat{\otimes}_{a} I\right)$ on $X \hat{\otimes}_{a} Y$.

(ii) Since $0 \in \rho(A)$ and $0 \epsilon \rho(B), A \otimes B$ maps $D[A] \otimes D[B]$ onto $X \otimes Y$, and by uniformness of $\alpha$ and by case (i) above we obtain $A^{-1} \hat{\otimes}_{\alpha} B^{-1}=$ $\left(A^{-1} \hat{\otimes}_{a} B\right)\left(I \hat{\otimes}_{a} B^{-1}\right)=\left(I \hat{\otimes}_{a} B^{-1}\right)\left(A^{-1} \hat{\otimes}_{a} I\right)$ on $X \hat{\otimes}_{a} Y$. Thus if $A \hat{\otimes}_{a} I$ and $I \hat{\otimes}_{a} B$ are arbitrary maximal extensions, then

$$
\begin{aligned}
D[(A \underset{\alpha}{\hat{\otimes}} I)(I \underset{\alpha}{\hat{\otimes}} B)] & \equiv(I \underset{a}{\hat{\otimes}} B)^{(-1)} H_{A \otimes I} \subset\left(I \underset{a}{\hat{\otimes}} B^{-1}\right) H_{A \otimes I} \\
& =\left(I \underset{\alpha}{\hat{\otimes}} B^{-1}\right) R\left[A^{-1} \underset{a}{\hat{\otimes}} I\right]=R\left[A^{-1} \underset{a}{\hat{\otimes}} B^{-1}\right]=H_{A \otimes 3}
\end{aligned}
$$

and similarly $D\left[\left(I \hat{\otimes}_{\alpha} B\right)\left(A \hat{\otimes}_{\alpha} I\right)\right] \subset H_{A \otimes B}$. This proves

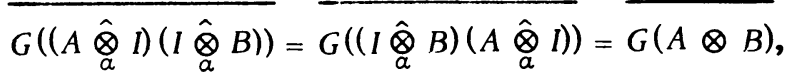


since $\left(A \hat{\otimes}_{\alpha} I\right)\left(I \hat{\otimes}_{\alpha} B\right)$ and $\left(I \hat{\otimes}_{\alpha} B\right)\left(A \hat{\otimes}_{\alpha} I\right)$ are extensions of $A \otimes B$.

To show the second assertion let $A \hat{\otimes}_{\alpha} B$ be an arbitrary maximal extension. First note that if $u \in D[A] \otimes D[B]$ then $(A \otimes B) u=(A \otimes I)(I \otimes B) u=$ $(I \otimes B)(A \otimes I) u$. Now let $u \in D\left[A \hat{\otimes}_{\alpha} B\right] \equiv H_{A \otimes B}$ but $u \notin D[A] \otimes D[B] ;$ there exists a sequence $\left\{u_{\nu}\right\}_{\nu=1}^{\infty}$ in $D[A] \otimes D[B]$ such that $\left(u_{\nu},(A \otimes B) u_{\nu}\right)$ converges to $\left(u, A \hat{\otimes}_{a} B u\right)$ in $\left(X \hat{\otimes}_{a} Y\right) \times\left(X \hat{\otimes}_{a} Y\right)$ as $\nu \rightarrow \infty$. Clearly $u_{\nu} \in D[I \otimes B] \equiv$ $X \otimes D[B]$ and by continuity $(I \otimes B) u_{\nu}=\left(A^{-1} \otimes I\right)(A \otimes B) u_{\nu}$ converges to $\left(A^{-1} \hat{\otimes}_{a} I\right)\left(A \hat{\otimes}_{a} B\right) u$ as $\nu \rightarrow \infty$, so that $u \in H_{I \otimes B}$. By Proposition 1.2 or 1.4, $u \notin X \otimes Y$ or $u \notin X \otimes D[B]$. Thus $D\left[A \hat{\otimes}_{\alpha} B\right] \backslash(D[A] \otimes D[B]) \subset H_{I \otimes B} \backslash(X \otimes D[B])$. There exists then a maximal extension $I \hat{\otimes}_{\alpha} B$ of $I \otimes B$ such that $I \hat{\otimes}_{\alpha} B u=$ $\left(A^{-1} \hat{\otimes}_{a} I\right)\left(A \hat{\otimes}_{a} B\right) u$ for $u \in D\left[A \hat{\otimes}_{a} B\right] \backslash(D[A] \otimes D[B])$. Then clearly $I \hat{\otimes}_{\alpha} B u$ $\epsilon R\left[A^{-1} \hat{\otimes}_{\alpha} I\right]=H_{A \otimes I}$ or $u \in\left(I \hat{\otimes}_{a} B\right)^{(-1)} H_{A \otimes I} I \otimes B$ maps $X \otimes D[B]$ onto $X \otimes Y$ and $I \hat{\otimes}_{\alpha} B$ as well as $I \otimes B$ is one-to-one (cf. [9, Corollary 4.2]), so, by Proposition 1.2 or $1.4, u \in D\left[A \hat{\otimes}_{\alpha} B\right] \backslash(D[A] \otimes D[B])$ implies $I \hat{\otimes}_{a} B u \epsilon$ $H_{A \otimes I} \backslash(D[A] \otimes Y)$. There exists a maximal extension $A \hat{\otimes}_{\alpha} I$ of $A \otimes I$ such that $\left(A \hat{\otimes}_{\alpha} I\right)\left(I \hat{\otimes}_{\alpha} B\right) u=A \hat{\otimes}_{\alpha} B u$ for $u \in D\left[A \hat{\otimes}_{\alpha} B\right] \backslash(D[A] \otimes D[B])$. In the same way we shall find suitable maximal extensions $A \hat{\otimes}_{a} I$ and $I \hat{\otimes}_{a} B$ for which $\left(I \hat{\otimes}_{\alpha} B\right)\left(A \hat{\otimes}_{\alpha} I\right)=A \hat{\otimes}_{\alpha} B$ holds.

(iii) We may assume $B$ and $B^{-1}$ are continuous on $Y$. Then $I \otimes B$ and $I \otimes B^{-1}$ are automorphisms of $X \otimes Y$, so that by uniformness of $\alpha$ there exists a positive constant $C$ such that, for $u \in D[A] \otimes Y$,

$$
C^{-1}\|(A \otimes I) u\|_{a} \leq\|(A \otimes B) u\|_{\alpha} \leq C\|(A \otimes I) u\|_{\alpha} .
$$

Hence $H_{A \otimes B}=H_{A \otimes I}$, and $\left(I \hat{\otimes}_{\alpha} B\right) H_{A \otimes I}=\left(I \hat{\otimes}_{\alpha} B^{-1}\right) H_{A \otimes I}=H_{A \otimes I}$. If $A \hat{\otimes}_{a} I$ is an arbitrary maximal extension, then

$$
D[(A \underset{\alpha}{\hat{\otimes}} I)(I \underset{a}{\hat{\otimes}} B)] \equiv(I \underset{\alpha}{\otimes} B)^{(-1)} H_{A \otimes I}=\left(I \underset{a}{\otimes} B^{-1}\right) H_{A \otimes I}=H_{A \otimes I}=H_{A \otimes B}
$$

and

$D[(I \underset{a}{\hat{\otimes}} B)(A \underset{a}{\hat{\otimes}} I)] \equiv(A \underset{\alpha}{\hat{\otimes}} I)^{(-1)} H_{I \otimes B}=(A \underset{a}{\hat{\otimes}} I)^{(-1)}(X \underset{a}{\hat{\otimes}} Y)=D[A \underset{\alpha}{\hat{\otimes}} I]=H_{A \otimes I}=H_{A \otimes B^{*}}$ It follows, though more stronger than the first assertion of Proposition 1.6, that $\left(A \hat{\otimes}_{\alpha} I\right)\left(I \hat{\otimes}_{\alpha} B\right)$ and $\left(I \hat{\otimes}_{\alpha} B\right)\left(A \hat{\otimes}_{\alpha} I\right)$ are maximal extensions of $A \otimes B$. In this case the second assertion is evident; in fact, if $A \hat{\otimes}_{\alpha} B$ is an arbitrary maximal extension, then $\left(A \hat{\otimes}_{\alpha} B\right)\left(I \hat{\otimes}_{\alpha} B\right)^{-1}=\left(A \hat{\otimes}_{\alpha} B\right)\left(I \hat{\otimes}_{\alpha} B^{-1}\right)$ and $\left(I \hat{\otimes}_{\alpha} B\right)^{-1}\left(A \hat{\otimes}_{\alpha} B\right)$ $=\left(I \hat{\otimes}_{a} B^{-1}\right)\left(A \hat{\otimes}_{a} B\right)$ are maximal extensions of $A \otimes I$.

Finally, if $\alpha$ is faithful so that $A \hat{\otimes}_{a} B, A \hat{\otimes}_{\alpha} I$ and $I \hat{\otimes}_{\alpha} B$ are closed [9, Theorem 3.8], then (1.7) is seen to be true for cases (i) and (iii) in our proof as above. For case (ii) equality holds valid in (1.8) since $A \hat{\otimes}_{\alpha} I$ and $I \hat{\otimes}_{\alpha} B$ map onto $X \hat{\otimes}_{\alpha} Y$, which yields (1.7). Q.E.D. 
Proposition 1.7. Let a be a uniform reasonable norm on $X \otimes Y$. Let $A$ : $D[A] \subset X \rightarrow X$ be a densely defined closed linear operator with nonempty resolvent set $\rho(A)$. Then for any maximal extension $A \hat{\otimes}_{a} I$ of $A \otimes I$ the closures of the graphs of $\left(A \hat{\otimes}_{\alpha} I\right)^{j}$ and $A^{j} \otimes I$ coincide.

If $\alpha$ is in addition faitbful, then ${ }^{*} A^{j} \hat{\otimes}_{\alpha} I=\left(A \hat{\otimes}_{\alpha} I\right)^{j}$.

Proof. Note $A^{j}$ is closed since $\rho(A) \neq \varnothing$.

First suppose $0 \in \rho(A)$. Then by uniformness of $\alpha, A^{-j} \hat{\otimes}_{\alpha} I=\left(A^{-1} \hat{\otimes}_{a} I\right)^{j}$ on $X \hat{\otimes}_{a} Y$. If $A \hat{\otimes}_{a} I$ is a maximal extension, then

$$
\begin{aligned}
D\left[(A \underset{a}{\hat{\otimes}} I)^{j}\right] & \equiv(A \underset{a}{\hat{\otimes}} I)^{(-1)} D\left[(A \underset{a}{\hat{\otimes}} I)^{j-1}\right] \\
& \subset\left(A^{-1} \underset{a}{\hat{\otimes}} I\right) D\left[(A \underset{a}{\hat{\otimes}} I)^{j-1}\right] \\
& \subset\left(A^{-1} \underset{a}{\hat{\otimes}} I\right)^{j-1} D[A \underset{a}{\hat{\otimes}} I]=\left(A^{-1} \underset{a}{\hat{\otimes}} I\right)^{j-1} H_{A \otimes I} \\
& =R\left[\left(A^{-1} \underset{a}{\hat{\otimes}} I\right)^{j}\right]=R\left[A^{-j} \underset{a}{\hat{\otimes}} I\right]=H_{A^{j}} .
\end{aligned}
$$

Now we remove the assumption $0 \in \rho(A)$. If $\lambda \in \rho(A)$, we have

$$
A^{j}=C_{1}(A ; j)(A-\lambda I)^{j} \text { and }(A-\lambda I)^{j}=C_{2}(A ; j) A^{j}+C_{3}(A ; j),
$$

where $C_{i}(A ; j), i=1,2,3$, are certain bounded operators on $X$ with $C_{i}(A ; j) A C$ $A C_{i}(A ; j)$. It follows by uniformness of $a$ that there exists a positive constant $C$ such that for all $u \in D\left[A^{j}\right] \otimes Y=D\left[(A-\lambda I)^{j}\right] \otimes Y$

$$
C^{-1}\left\|\left((A-\lambda I)^{j} \otimes I\right) u\right\|_{a} \leq\left\|\left(A^{j} \otimes I\right) u\right\|_{a}+\|u\|_{a} \leq C\left\|\left((A-\lambda I)^{j} \otimes I\right) u\right\|_{a},
$$

whence $H_{A j \otimes I}=H_{(A-\lambda I)^{j \otimes I}}$. On the other hand $(A-\lambda I) \otimes I=A \otimes I-\lambda I \otimes I$.

Hence $(A-\lambda I) \hat{\otimes}_{a} I=A \hat{\otimes}_{a} I-\lambda I \hat{\otimes}_{\alpha} I$; by this is meant that any maximal extension $(A-\lambda I) \hat{\otimes}_{a} I$ of $(A-\lambda I) \otimes I$ equals $A \hat{\otimes}_{a} I-\lambda I \hat{\otimes}_{\alpha} I$ for some maximal extension $A \hat{\otimes}_{a} I$ of $A \otimes I$, and vice versa. It follows that $D\left[\left(A \hat{\otimes}_{a} I\right)^{j}\right]=$ $D\left[\left(A \hat{\otimes}_{a} I-\lambda I \hat{\otimes}_{a} I\right)^{j}\right]=D\left[\left((A-\lambda I) \hat{\otimes}_{a} I\right)^{j}\right]$. From the preceding case, we obtain $D\left[\left(A \hat{\otimes}_{a} I\right)^{j}\right] \subset H_{(A-\lambda I)^{j \otimes I}}=H_{A j \otimes I^{*}}$ This proves

since $\left(A \hat{\otimes}_{\alpha} I\right)^{j} \supset A^{j} \otimes I$.

$$
\overline{G\left((A \underset{a}{\hat{\otimes}} I)^{j}\right)}=\overline{G\left(A^{j} \otimes I\right)}
$$

The assertion for faithful $a$ is evident, since the closure of a closable operator is its unique maximal extension. Q.E.D.

From Propositions 1.6 and 1.7 follows immediately

Theorem 1.8. Let a be a uniform reasonable norm on $X \otimes Y$. Let $A$ and $B$ be densely defined closed linear operators with nonempty resolvent sets $\rho(A)$, 
$\rho(B)$ such that it does not occur that one of the extended spectra $\sigma_{e}(A)$ and $\sigma_{e}(B)$ contains 0 while the other contains $\infty$. Then for any maximal extensions $A \hat{\otimes}_{\alpha} I$ of $A \otimes I$ and $I \hat{\otimes}_{\alpha} B$ of $I \otimes B$, the closures of the graphs of $\left(A \hat{\otimes}_{\alpha} I\right)^{j}\left(I \hat{\otimes}_{\alpha} B\right)^{k}$, $\left(I \hat{\otimes}_{\alpha} B\right)^{k}\left(A \hat{\otimes}_{a} I\right)^{j}$ and $A^{j} \otimes B^{k}$ coincide for eacb pair $(j, k)$ of nonnegative integers.

If $\alpha$ is in addition faithful so that the $A^{j} \hat{\otimes}_{\alpha} B^{k}$ are closed in $X \hat{\otimes}_{\alpha} Y$, we bave

$$
A^{j} \underset{a}{\hat{\otimes}} B^{k}=(A \underset{a}{\hat{\otimes}} I)^{j}(I \underset{a}{\hat{\otimes}} B)^{k}=(I \underset{a}{\hat{\otimes}} B)^{k}(A \underset{a}{\hat{\otimes}} I)^{j},
$$

so that for polynomials of the form (1.1)

$$
\sum c_{j k} A^{j} \underset{\alpha}{\hat{\otimes}} B^{k}=P(A \underset{\alpha}{\hat{\otimes}} I, I \underset{\alpha}{\hat{\otimes}} B) .
$$

Corollary 1.9. Under the same condition as in Theorem 1.8 we bave

$$
\begin{aligned}
& \left(A^{j} \underset{a}{\hat{\otimes}} B^{k}\right)^{\prime}=\left((A \underset{a}{\hat{\otimes}} I)^{j}(I \underset{a}{\hat{\otimes}} B)^{k}\right)^{\prime}=\left((I \underset{a}{\hat{\otimes}} B)^{k}(A \underset{a}{\hat{\otimes}} I)^{j}\right)^{\prime}, \\
& \left(A^{j} \underset{a}{\hat{\otimes}} B^{k}\right)^{\prime}=\left((A \underset{a}{\hat{\otimes}} I)^{\prime}\right)^{j}\left((I \underset{a}{\hat{\otimes}} B)^{\prime}\right)^{k}=\left((I \underset{a}{\hat{\otimes}} B)^{\prime}\right)^{k}\left((A \underset{a}{\hat{\otimes}} I)^{\prime}\right)^{j} .
\end{aligned}
$$

Proof. (1.10) is a direct consequence of Theorem 1.8. To prove (1.11) set $A \hat{\otimes}_{a} I=\mathbf{A}$ and $I \hat{\otimes}_{\alpha} B=\mathbf{B}$. From $(1.10)$ we obtain $\left(A^{j} \hat{\otimes}_{a} B^{k}\right)^{\prime} \supset\left(\mathbf{A}^{\prime}\right)^{j}\left(\mathbf{B}^{\prime}\right)^{k}$ and $\left(A^{j} \hat{\otimes}_{a} B^{k}\right)^{\prime} \supset\left(\mathbf{B}^{\prime}\right)^{k}\left(\mathbf{A}^{\prime}\right)^{j}$. Note $\left(\mathbf{A}^{\prime}\right)^{j}\left(\mathbf{B}^{\prime}\right)^{k}$ and $\left(\mathbf{B}^{\prime}\right)^{k}\left(\mathbf{A}^{\prime}\right)^{j}$ are closed in $\left(X \hat{\otimes}_{a} Y\right)^{\prime}$. In fact, since the adjoints $A^{\prime}$ and $B^{\prime}$ are closed and $\rho\left(A^{\prime}\right)=\rho(A)=$ $\rho\left(A \hat{\otimes}_{a} I\right)=\rho(A) \neq \varnothing$ and $\rho\left(B^{\prime}\right)=\rho(B)=\rho\left(I \hat{\otimes}_{a} B\right)=\rho(B) \neq \emptyset[9$, Corollary 4.2], $\left(\mathbf{A}^{\prime}\right)^{j}$ and $\left(\mathbf{B}^{\prime}\right)^{k}$ are closed. Observing the equivalent condition reformulated in Remark 1.3, we obtain the inequalities

$$
\left\|\left(\mathbf{B}^{\prime}\right)^{k} u^{\prime}\right\|_{a^{\prime}} \leq C_{A}\left[\left\|\left(\mathbf{A}^{\prime}\right)^{j}\left(\mathbf{B}^{\prime}\right)^{k} u^{\prime}\right\|_{a^{\prime}}+\left\|u^{\prime}\right\|_{a^{\prime}}\right]
$$

for $u^{\prime} \in D\left[\left(\mathbf{A}^{\prime}\right)^{j}\left(\mathbf{B}^{\prime}\right)^{k}\right]$, and

$$
\left\|\left(\mathrm{A}^{\prime}\right)^{j} u^{\prime}\right\|_{\alpha^{\prime}} \leq C_{B}\left[\left\|\left(\mathbf{B}^{\prime}\right)^{k}\left(\mathbf{A}^{\prime}\right)^{j} u^{\prime}\right\|_{a^{\prime}}+\left\|u^{\prime}\right\|_{a^{\prime}}\right]
$$

for $u^{\prime} \in D\left[\left(\mathbf{B}^{\prime}\right)^{k}\left(\mathbf{A}^{\prime}\right)^{j}\right]$, where $C_{A}$ and $C_{B}$ are constants independent of $u^{\prime}$. Then the closedness of $\left(\mathbf{A}^{\prime}\right)^{j}\left(\mathbf{B}^{\prime}\right)^{k}$ and $\left(\mathbf{B}^{\prime}\right)^{k}\left(\mathbf{A}^{\prime}\right)^{j}$ follows in virtue of the Banach-Schauder theorem.

Now (1.11) is clear for case (i). The condition (ii) implies (with the aid of Theorem 4.13 in [9] $0 \notin \sigma(A)^{j} \sigma(B)^{k}=\sigma\left(A^{j}\right) \sigma\left(B^{k}\right)=\sigma\left(A^{j} \hat{\otimes}_{\alpha} B^{k}\right)=\sigma\left(\left(A^{j} \hat{\otimes}_{\alpha} B^{k}\right)^{\prime}\right)$, that is, $\left(A^{j} \hat{\otimes}_{\alpha} B^{k}\right)^{\prime}$ maps onto $\left(X \hat{\otimes}_{\alpha} Y\right)^{\prime}$ and has a continuous inverse. $\left(\mathbf{A}^{\prime}\right)^{j}\left(\mathbf{B}^{\prime}\right)^{k}$ has also the same property. Consequently $\left(A^{j} \hat{\otimes}_{a} B^{k}\right)^{\prime}=\left(A^{\prime}\right)^{j}\left(B^{\prime}\right)^{k}$. Similarly $\left(A^{j} \hat{\otimes}_{a} B^{k}\right)^{\prime}=\left(\mathbf{B}^{\prime}\right)^{k}\left(\mathbf{A}^{\prime}\right)^{j}$. For case (iii), we may assume $B$ and $B^{-1}$ are continuous on $Y$. Since $I \hat{\otimes}_{a} B^{k}=\left(I \hat{\otimes}_{a} B\right)^{k}$ is an automorphism of $X \hat{\otimes}_{a} Y$ with $I \hat{\otimes}_{a} B^{k} H_{A j \otimes I}=H_{A j \otimes I}$, we have only to show $\left(A^{j} \hat{\otimes}_{a} I\right)^{\prime}=\left(\left(A \hat{\otimes}_{a} I\right)^{\prime}\right)^{j}$ if $A$ is densely defined and closed with $\rho(A) \neq \emptyset$. But this follows from Proposition 1.7, 
since the adjoints of two densely defined linear operators whose graphs have the same closure coincide. Q.E.D.

2. Spectral mapping theorem. Developing an operational calculus for tensor products of linear operators, we have shown in [10] the spectral mapping theorem for certain polynomial operators of the kind (1.2). However, it is not applicable to the operator such as $A \otimes I+I \otimes B$ unless at least one of $A$ and $B$ is bounded.

We shall now enlarge the class of polynomials for which the spectral mapping theorem holds. In this section only polynomial operators of the kind (1.2) are considered.

Given $n$ subsets $G_{j}$ of $\mathrm{C}, 1 \leq j \leq n$, and a polynomial $P\left(\zeta_{1}, \cdots, \zeta_{n}\right)$, we can define $P\left(G_{1}, \cdots, G_{n}\right)$ in an obvious way if $P\left(\zeta_{1}, \cdots, \zeta_{n}\right)$ is independent of those variables $\zeta_{j}$ for which the $G_{j}$ are empty, and otherwise we set $P\left(G_{1}, \cdots, G_{n}\right)=\varnothing$.

In the sequel, $A$ and $B$ are assumed to be densely defined closed linear operators with nonempty resolvent sets $\rho(A), \rho(B)$. For $R>0, K(0 ; R)$ will denote the closed disc $\{\zeta ;|\zeta| \leq R\}$.

Let $\mathcal{P}(A, B)$ be the class of polynomials $P(\xi, \eta)$ in $\xi$ and $\eta$ having the following property: for any open neighbourhood $W$ in $\mathbf{C}$ of $P(\sigma(A), \sigma(B)$ ) (when at least one of $\sigma(A)$ and $\sigma(B)$ is empty take $W=\mathcal{C} K(0 ; R)$ for any $R>0)$, there exists a nonempty open set $U$ (resp. $V$ ) whose complement $\mathcal{C} U$ (resp. $\mathcal{C} V$ ) is contained in $\rho(A)$ (resp. $\rho(B)$ ) and whose boundary $\partial U$ (resp. $\partial V$ ) within the closed disc $K(0 ; R)$ for each $R>0$ consists of a finite number of rectifiable Jordan arcs and has a length $O(R)$ as $R \rightarrow \infty$ such that

(i) $P(U, V) \subset W$; in $\overline{\mathcal{C}} V$;

(ii) $\xi R(\xi ; A)$ is uniformly bounded in $\mathcal{C} U$ and $\eta R(\eta ; B)$ uniformly bounded

(iii) $P(\xi, \eta)(|\xi|+|\eta|)^{-1}$ is bounded away from zero on $\bar{U} \times \bar{V}$ for sufficiently large $|\xi|+|\eta|$.

Condition (i) means roughly that $P(\xi, \eta)$ is, as a mapping of $\mathbf{C}^{2}$ into $\mathbf{C}$, continuous at $\sigma(A) \times \sigma(B)$ (cf. [10]). Condition (iii) implies that $P(\sigma(A), \sigma(B))$ is closed in $\mathbf{C}$.

Then

Theorem 2.1. For $P \in \mathscr{P}(A, B)$

$$
P(\sigma(A), \sigma(B))=\sigma(P(A \otimes I, I \otimes B))=\sigma(\tilde{P}(A \otimes I, I \otimes B))
$$

bolds independently of a given uniform reasonable norm $\alpha$ on $X \otimes Y$. This means that (2.1) bolds if $\sigma(A) \neq \varnothing$ and $\sigma(B) \neq \varnothing$, and further that the spectra of $P(A \otimes I, I \otimes B)$ and its maximal extension $\tilde{P}(A \otimes I, I \otimes B)$ are empty if and 
only if at least one of $\sigma(A)$ and $\sigma(B)$ is empty.

To prove Theorem 2.1 we shall need the following lemmas.

Lemma 2.2. Let $T: D[T] \subset E \rightarrow E$ be a linear operator in a Banach space $E, G(T)$ its graph and $K_{T}$ the projection of the closure $\overline{G(T)} \subset E \times E$ into the

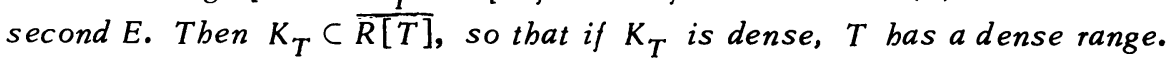

Proof. Obvious.

Lemma 2.3. If $T: D[T] \subset E \rightarrow E$ is a densely defined closed linear operator in a Banach space $E$ with nonempty resolvent set $\rho(T)$, then $D\left[T^{n+m}\right]$ is dense in $D\left[T^{n}\right]$ with respect to the graph norm of $T^{n}$ or, equivalently, to the norm $\|x\|+\sum_{j=1}^{n}\left\|T^{j} x\right\|$ for any positive integer $m$.

Proof. Note for each positive integer $n, D\left[T^{n}\right]$ is by the Hahn-Banach theorem dense in $E$. Thus $(T-\lambda I)^{n}$ for $\lambda \epsilon \rho(T)$ is a densely defined closed linear operator of $D\left[T^{n}\right] \subset E$ onto $E$ with bounded inverse $(T-\lambda I)^{-n}$. Obvious is the equivalence of the graph norms of $T^{n}$ and $(T-\lambda I)^{n} . D\left[T^{n+m}\right]$ is dense in $D\left[T^{n}\right]$ with respect to the graph norm of $(T-\lambda I)^{n}$ if and only if the closure of the restriction of $(T-\lambda I)^{n}$ to $D\left[T^{n+m}\right]$ coincides with $(T-\lambda I)^{n}$ if and only if $(T-\lambda I)^{n} D\left[T^{n+m}\right]$ is dense in $E$. But the last statement is obviously true, since $D\left[T^{m}\right]=(T-\lambda I)^{n} D\left[T^{n+m}\right]$ is dense in $E$ for $m \geq 1$. Q.E.D.

Proof of Theorem 2.1. We may assume that $P(\xi, \eta)$ is not identically constant, and is a polynomial of degrees $m$ in $\xi$ and $n$ in $\eta$ of the form (1.1). The last equality follows from the fact that the spectrum is unchanged under maximal extensions [9].

We shall be able to show the inclusion $P(\sigma(A), \sigma(B)) \subset \sigma(P(A \otimes I, I \otimes B))$ in the same way as in [9], by making the most of the fact that the spectrum is divided into the approximate point spectrum which is closed in $\mathbf{C}$, and its complement which is open in $\mathrm{C}$. We denote the approximate point spectra of $A$ and $B$ by $\pi(A), \pi(B)$, respectively. Let $(\alpha, \beta) \in \sigma(A) \times \sigma(B)$. We have

$$
\begin{aligned}
P(A \otimes I, I \otimes B) & P(\alpha, \beta)(I \otimes I) \\
& =P_{1}(A \otimes I, I \otimes B)\{(A-\alpha I) \otimes I\}+P_{2}(A \otimes I, I \otimes B)\{I \otimes(B-\beta I)\},
\end{aligned}
$$

where $P_{1}(\xi, \eta)$ (resp. $\left.P_{2}(\xi, \eta)\right)$ is a polynomial of degrees $\leq m-1$ (resp. $m$ ) in $\xi$ and $\leq n$ (resp. $n-1$ ) in $\eta$. To treat the case $(\alpha, \beta) \in \pi(A) \times \pi(B)$ and the case $(\alpha, \beta) \in(\sigma(A) \backslash \pi(A)) \times(\sigma(B) \backslash \pi(B))$, note that if $\lambda \in \pi(A)$ there exists a sequence $\left\{x_{\nu}\right\}_{\nu=1}^{\infty} \subset D\left[A^{m}\right]$ of unit vectors such that for any polynomial $S(\xi)$ of degree $\leq m$ the sequence $\left\{S(A) x_{\nu}\right\}$ is bounded and such that, for each polynomial $Q(\xi)$ of degree $\leq m-1, Q(A)(A-\lambda I) x_{\nu}$ converges to zero as $\nu \rightarrow \infty$, and that the same with $A, m$ replaced by $B, n$, respectively, is true. To treat 
the remaining case $(\alpha, \beta) \in \pi(A) \times(\sigma(B) \backslash \pi(B))$ or $(\alpha, \beta) \in(\sigma(A) \backslash \pi(A)) \times \pi(B)$, use the fact that if one of the open sets $G_{1}$ and $G_{2}$ in $C$ has an infinite number of boundary points and if a polynomial $P(\xi, \eta)$ has a zero $\left(\xi_{0}, \eta_{0}\right)$ in $G_{1} \times G_{2}$, then there exists a contour from $\left(\xi_{0}, \eta_{0}\right)$ to a boundary point of $G_{1} \times G_{2}$ lying in the set $\left\{(\xi, \eta) \in \bar{G}_{1} \times \bar{G}_{2} ; P(\xi, \eta)=0\right\}$. (For the details see [10].)

We show now the reverse inclusion, assuming $P(\sigma(A), \sigma(B)) \neq \mathbf{C}$. Let $\lambda \notin$ $P(\sigma(A), \sigma(B))$. If $\sigma(A)$ or $\sigma(B)$ is empty, $\lambda$ shall be an arbitrary complex number. $P \in \mathcal{P}(A, B)$. By assumption there exist nonempty open sets $U$ and $V$ with $\mathcal{C}_{U} \subset \rho(A)$ and $\mathcal{C}_{V} \subset \rho(B)$ satisfying (ii) such that (i) $|P(\xi, \eta)-\lambda|$ is bounded away from zero on $\bar{U} \times \bar{V}$ and such that (iii) $|P(\xi, \eta)-\lambda|(|\xi|+|\eta|)^{-1}$ is bounded away from zero on $\bar{U} \times \bar{V}$ for sufficiently large $|\xi|+|\eta|$.

Then for $v \in X \otimes Y$ the integral along the oriented contours $\partial U$ and $\partial V$

$$
(2 \pi i)^{-2} \int_{\partial U} \int_{\partial V}(P(\xi, \eta)-\lambda)^{-1}[R(\xi ; A) \otimes R(\eta ; B)] v d \xi d \eta
$$

exists as an element of the Banach space $X \hat{\otimes}_{a} Y$ and defines on account of (ii) and (iii)' a continuous linear operator of $X \otimes Y \subset X \hat{\otimes}_{a} Y$ into $X \hat{\otimes}_{\alpha} Y$, since $a$ is uniform. Its continuous extension to the entire space $X \hat{\otimes}_{a} Y$ will be denoted by $\widetilde{P_{\lambda}^{-1}}(A \otimes I, I \otimes B)$ with $P_{\lambda}=P-\lambda\left(\right.$ cf. $\Theta\left(\widetilde{P_{\lambda}^{-1}} ; A, B\right)$ in the notation of $[10])$. We shall show each maximal extension $\tilde{P}(A \otimes I, I \otimes B)-\lambda I \hat{\otimes}_{a} I$ of $P(A \otimes I, I \otimes B)-\lambda I \otimes I$ has a densely defined continuous inverse.

For $u \in D\left[A^{2 m}\right] \otimes D\left[B^{2 n}\right]$,

$$
\begin{aligned}
\widetilde{P_{\lambda}^{-1}}(A \otimes I, & I \otimes B)[\widetilde{P}(A \otimes I, I \otimes B)-\lambda I \underset{a}{\hat{\otimes}} I] u \\
= & \widetilde{P_{\lambda}^{-1}(A \otimes I, I \otimes B)[P(A \otimes I, I \otimes B)-\lambda I \otimes I] u} \\
= & (2 \pi i)^{-2} \int_{\partial U} \int_{\partial V}(P(\xi, \eta)-\lambda)^{-1}[R(\xi ; A) \otimes R(\eta ; B)] \\
& \cdot[P(A \otimes I, I \otimes B)-\lambda I \otimes I] u d \xi d \eta .
\end{aligned}
$$

The following identities are easily verified:

$$
\begin{aligned}
& R(\xi ; A) x=\sum_{j=1}^{m}\left(\xi-\xi_{0}\right)^{-j}\left(A-\xi_{0} I\right)^{j-1} x+\left(\xi-\xi_{0}\right)^{-m} R(\xi ; A)\left(A-\xi_{0} I\right)^{m} x \\
& R(\eta ; B) y=\sum_{k=1}^{n}\left(\eta-\eta_{0}\right)^{-k}\left(B-\eta_{0} I\right)^{k-1} y+\left(\eta-\eta_{0}\right)^{-n} R(\eta ; B)\left(B-\eta_{0} I\right)^{n} y
\end{aligned}
$$

for $(x, y) \in D\left[A^{m}\right] \times D\left[B^{n}\right]$.

For $\xi_{0} \notin U$ and $\eta_{0} \notin V$ utilizing the identities (2.3) and (2.4) we obtain by the Cauchy integral theorem 


$$
\begin{gathered}
\widetilde{P_{\lambda}^{-1}}(A \otimes I, I \otimes B)\left[\tilde{P}(A \otimes I, I \otimes B)-\lambda I \underset{a}{\left.\hat{\otimes}_{a} I\right] u}\right. \\
=(2 \pi i)^{-2} \int_{\partial U} \int_{\partial V}(P(\xi, \eta)-\lambda)^{-1}\left(\xi-\xi_{0}\right)^{-m}\left(\eta-\eta_{0}\right)^{-n}[R(\xi ; A) \otimes R(\eta ; B)] \\
\cdot\left[\left(A-\xi_{0} I\right)^{m} \otimes\left(B-\eta_{0} I\right)^{n}\right][P(A \otimes I, I \otimes B)-\lambda I \otimes I] u d \xi d \eta \\
=(2 \pi i)^{-2} \int_{\partial U} \int_{\partial V}\left(\xi-\xi_{0}\right)^{-m}\left(\eta-\eta_{0}\right)^{-n}\left[\left(A-\xi_{0} I\right)^{m} \otimes\left(B-\eta_{0} I\right)^{n}\right] \\
\cdot[R(\xi ; A) \otimes R(\eta ; B)] u d \xi d \eta \\
+(2 \pi i)^{-2} \int_{\partial U} \int_{\partial V}(P(\xi, \eta)-\lambda)^{-1}\left(\xi-\xi_{0}\right)^{-m}\left(\eta-\eta_{0}\right)^{-n} \\
\cdot\left[\left(A-\xi_{0} I\right)^{m} \otimes\left(B-\eta_{0} I\right)^{n}\right][R(\xi ; A) \otimes R(\eta ; B)] \\
\cdot[P(A \otimes I, I \otimes B)-P(\xi, \eta) I \otimes I] u d \xi d \eta
\end{gathered}
$$

for the second integral vanishes if we note

$$
P(A \otimes I, I \otimes B)-P(\xi, \eta) I \otimes I=\sum_{j+k>0} b_{j k}(\xi I-A)^{j} \otimes(\eta I-B)^{k} .
$$

In virtue of Lemma 2.3, any $x \in D\left[A^{m}\right]$ can be approximated by a sequence in $D\left[A^{2 m}\right]$ with respect to the norm $\|x\|+\sum_{j=1}^{m}\left\|A^{j} x\right\|$ and the same is possible for $B$. Consequently, $D\left[A^{2 m}\right] \otimes D\left[B^{2 n}\right]$ is by the reasonableness of a dense in $D[P(A \otimes I, I \otimes B)]=D\left[A^{m}\right] \otimes D\left[B^{n}\right]$ with respect to the graph norm of $P(A \otimes I, I \otimes B)$ in $X \hat{\otimes}_{\alpha} Y$. Thus

$$
\widetilde{P_{\bar{\lambda}}^{-1}}(A \otimes I, I \otimes B)[\tilde{P}(A \otimes I, I \otimes B)-\lambda I \underset{\alpha}{\hat{\otimes} I} I] u=u
$$

is valid for all $u$ in the domain of $P(A \otimes I, I \otimes B)$ and so by continuity of ${\widetilde{P_{\lambda}^{-1}}}^{-1}(A \otimes I, I \otimes B)$ for all $u$ in the domain of any maximal extension $\widetilde{P}(A \otimes I, I \otimes B)$. This proves that the restriction of $\widetilde{P_{\lambda}^{-1}}(A \otimes I, I \otimes B)$ to the range $R\left[\tilde{P}(A \otimes I, I \otimes B)-\lambda I \hat{\otimes}_{\alpha} I\right]$ is a continuous inverse of $\tilde{P}(A \otimes I, I \otimes B)-$ $\lambda I \hat{\otimes}_{\alpha} I$. It remains to show the range of $\widetilde{P}(A \otimes I, I \otimes B)-\lambda I \hat{\otimes}_{\alpha} I$ is dense in $X \hat{\otimes}_{\alpha} Y$.

By the definition of the improper Riemann integral, for $v \in D\left[A^{2 m}\right] \otimes D\left[B^{2 n}\right]$ the integral (2.2), i.e. $u \equiv \widetilde{P_{\lambda}^{-1}}(A \otimes I, I \otimes B) v$, can be approximated by a sequence $\left\{u_{\nu}\right\}_{\nu=1}^{\infty}$ in $D\left[A^{2 m+1}\right] \otimes D\left[B^{2 n+1}\right]$ of Riemann sums. Obvious is that $[P(A \otimes I, I \otimes B)-\lambda I \otimes I] u_{\nu}$ is convergent to $v$ as $\nu \rightarrow \infty$, so that $u \equiv$ $\widetilde{P_{\lambda}^{-1}}(A \otimes I, I \otimes B) v$ belongs to $D[\tilde{P}(A \otimes I, I \otimes B)]$ by the definition of maximal extensions, that is, $\left(\widetilde{P_{\lambda}^{-1}}(A \otimes I, I \otimes B) v, v\right)$ for every $v \in D\left[A^{2 m}\right] \otimes D\left[B^{2 n}\right]$ belongs to the closure of the graph of $P(A \otimes I, I \otimes B)-\lambda I \otimes I$. Since $D\left[A^{2 m}\right] \otimes D\left[B^{2 n}\right]$ is dense in $X \hat{\otimes}_{a} Y$, it follows by Lemma 2.2 that $\tilde{P}(A \otimes I, I \otimes B)-\lambda I \hat{\otimes}_{\alpha} I$ as well as $P(A \otimes I, I \otimes B)-\lambda I \otimes I$ has a dense range. Q.E.D.

Remark 2.4. Assumption of the uniformness of the norm $\alpha$ in Theorem 2.1 is only used to assure that the integral (2.2) defines a continuous operator on $X \hat{\otimes}_{a} Y$ (cf. [9, Remark 4.10]). Theorem 2.1 is also valid for any reasonable norm 
$\alpha$ on $X \otimes Y$ for which if $u \in X \otimes Y$,

$$
\|[R(\xi ; A) \otimes R(\eta ; B)] u\|_{a} \leq\|R(\xi ; A)\|\|R(\eta ; B)\|\|u\|_{a}
$$

or

$$
\|[R(\xi ; A) \otimes R(\eta ; B)] u\|_{a} \leq \text { Constant } \cdot|\xi|^{-1}|\eta|^{-1}\|u\|_{a}
$$

on $\mathcal{C U}_{\times} \mathcal{C}_{V}$.

3. Closures of polynomial operators. If $\alpha$ is faithful and reasonable, we have seen in virtue of Theorem 1.1 that the polynomial operators (1.2) and (1.3) are closable in $X \hat{\otimes}_{\alpha} Y$. We shall now show these two closures coincide for faithful uniform reasonable $\alpha$, which reduces the spectral mapping theorem for the polynomial operators of the kind (1.2) to that for the polynomial operators of the kind (1.3).

Theorem 3.1. Let a be a faithful uniform reasonable norm on $X \otimes Y$ and $P \in \mathcal{P}(A, B)$ of the form (1.1). If $P(\sigma(A), \sigma(B)) \neq \mathbf{C}$, then

$$
\tilde{P}(A \otimes I, I \otimes B)=\left(\sum c_{j k} A^{j} \underset{a}{\hat{\otimes}} B^{k}\right)^{\sim}
$$

Proof. Since the right member of (3.1) is a closed extension of the left member, it suffices to show $\left(\sum c_{j k} A^{j} \hat{\otimes}_{a} B^{k}\right)^{\sim} \subset \widetilde{P}(A \otimes I, \underset{\sim}{l} \otimes B)$.

Let $\lambda \notin P(\sigma(A), \sigma(B))$. By Theorem 2.1 the closure $\tilde{P}(A \otimes I, I \otimes B)-$ $\lambda I \hat{\otimes}_{\alpha} I$ has a bounded inverse and maps onto $X \hat{\otimes}_{\alpha} Y$. Therefore we have only to show that $\left(\sum c_{j k} A^{j} \hat{\otimes}_{a} B^{k}\right)^{\sim}-\lambda I \hat{\otimes}_{a} I=\left(\sum c_{j k} A^{j} \hat{\otimes}_{a} B^{k}-\lambda I \hat{\otimes}_{a} I\right)^{\sim}$ is one-to-one, or that its adjoint has a dense range in $\left(X \hat{\otimes}_{\alpha} Y\right)^{\prime}$ with respect to the weak topology defined by the dual pair $\left\langle\left(X \hat{\otimes}_{a} Y\right)^{\prime}, D\left[\left(\sum c_{j k} A^{j} \hat{\otimes}_{a} B^{k}\right)^{\sim}\right]\right\rangle$ (cf. [14]). Since a densely defined closable operator and its closure have the same adjoint, we obtain similarly to (1.4)

$$
\begin{aligned}
{\left[\left(\sum c_{j k} A^{j} \underset{a}{\hat{\otimes}} B^{k}\right)^{\sim}-\lambda I \underset{a}{\otimes} I\right]^{\prime} } & =\left(\sum c_{j k} A^{j} \underset{a}{\hat{\otimes}} B^{k}-\lambda I \underset{a}{\otimes} I\right)^{\prime} \\
& \supset \sum c_{j k}\left(A^{\prime}\right)^{j} \otimes\left(B^{\prime}\right)^{k}-\lambda I^{\prime} \otimes I^{\prime} \\
& =P\left(A^{\prime} \otimes I^{\prime}, I^{\prime} \otimes B^{\prime}\right)-\lambda I^{\prime} \otimes I^{\prime} .
\end{aligned}
$$

We know $\sigma\left(A^{\prime}\right)=\sigma(A)$ and $\sigma\left(B^{\prime}\right)=\sigma(B)$, so $\lambda \notin P(\sigma(A), \sigma(B))=$ $P\left(\sigma\left(A^{\prime}\right), \sigma\left(B^{\prime}\right)\right)$, and $R\left(\xi ; A^{\prime}\right) \equiv\left(\xi I^{\prime}-A^{\prime}\right)^{-1}=\left((\xi I-A)^{-1}\right)^{\prime} \equiv R(\xi ; A)^{\prime}$, $R\left(\eta ; B^{\prime}\right) \equiv\left(\eta I^{\prime}-B^{\prime}\right)^{-1}=\left((\eta I-B)^{-1}\right)^{\prime} \equiv R(\eta ; B)^{\prime}$. Therefore condition (ii) implies that $\xi R\left(\xi ; A^{\prime}\right)$ is bounded in $\mathcal{C}_{J}$ and $\eta R\left(\eta ; B^{\prime}\right)$ bounded in $\mathcal{C}_{V}$. We can define then in the same way as in the proof of Theorem $2.1{P_{\lambda}^{-1}}_{\left(A^{\prime} \otimes I^{\prime}, I^{\prime} \otimes B^{\prime}\right)}$ with $P_{\lambda}=P-\lambda$ by means of the integral $(2.2)$ with $R(\xi ; A)$ replaced by $R\left(\xi ; A^{\prime}\right)$ and $R(\eta ; B)$ by $R\left(\eta ; B^{\prime}\right)$. The closure $\widetilde{P}\left(A^{\prime} \otimes I^{\prime}, I^{\prime} \otimes B^{\prime}\right)-\lambda I^{\prime} \hat{\otimes}_{a^{\prime}} I^{\prime}$ of $P\left(A^{\prime} \otimes I^{\prime}, I^{\prime} \otimes B^{\prime}\right)-\lambda I^{\prime} \otimes I^{\prime}$ in $X^{\prime} \hat{\otimes}_{\alpha^{\prime}} Y^{\prime}$ has a continuous inverse which is 
the restriction of $\widetilde{P_{\lambda}^{-1}}\left(A^{\prime} \otimes I^{\prime}, I^{\prime} \otimes B^{\prime}\right)$ to the range $R\left[\widetilde{P}\left(A^{\prime} \otimes I^{\prime}, I^{\prime} \otimes B^{\prime}\right)-\lambda I^{\prime} \hat{\otimes}_{a} I^{\prime}\right]$. Note $\widetilde{P}\left(A^{\prime} \otimes I^{\prime}, I^{\prime} \otimes B^{\prime}\right)-\lambda I^{\prime} \hat{\otimes}_{a^{\prime}} I^{\prime}$ is closed by (3.2) or because the dual norm $\alpha^{\prime}$ is a faithful uniform reasonable norm on $X^{\prime} \otimes Y^{\prime}$.

The considerations as before show that the range $R\left[\tilde{P}\left(A^{\prime} \otimes I^{\prime}, I^{\prime} \otimes B^{\prime}\right)-\lambda I^{\prime} \hat{\otimes}_{\alpha^{\prime}} I^{\prime}\right]$ contains $D\left[\left(A^{\prime}\right)^{2 m}\right] \otimes D\left[\left(B^{\prime}\right)^{2 n}\right]$. Similarly to the proof of Theorem 1.1 the faithfulness of a yields that $D\left[\left(A^{\prime}\right)^{2 m}\right] \otimes D\left[\left(B^{\prime}\right)^{2 n}\right]$ is dense in $X^{\prime} \hat{\otimes}_{a^{\prime}} Y^{\prime}$ and also in $\left(X \hat{\otimes}_{a} Y\right)^{\prime}$ with respect to the weak topology defined by the dual pair $\left\langle\left(X \hat{\otimes}_{a} Y\right)^{\prime}, X \hat{\otimes}_{a} Y\right\rangle$. Thus the range of $\tilde{P}\left(A^{\prime} \otimes I^{\prime}, I^{\prime} \otimes . B^{\prime}\right)-\lambda I^{\prime} \hat{\otimes}_{a^{\prime}} I^{\prime}$ is dense in $\left(X \hat{\otimes}_{\alpha} Y\right)^{\prime}$ in the same weak topology and so in the weak topology defined by the dual pair $\left\langle\left(X \hat{\otimes}_{a} Y\right)^{\prime}, D\left[\left(\sum c_{j k} A^{j} \hat{\otimes}_{a} B^{k}\right)^{\sim}\right]\right\rangle$. Q.E.D.

Since the spectrum is unchanged under the closure operation, the following theorem is a direct consequence of Theorems 2.1 and 3.1. Note $\sigma\left(A \hat{\otimes}_{a} I\right)=\sigma(A)$ and $\sigma\left(I \hat{\otimes}_{\alpha} B\right)=\sigma(B)$.

Theorem 3.2. If $a$ is a faithful uniform reasonable norm on $X \otimes Y$, then for $P \in \mathcal{P}(A, B)$ of the form (1.1)

$$
\begin{aligned}
P(\sigma(A), \sigma(B)) & =P(\sigma(A \underset{a}{\otimes} I), \sigma(I \underset{\alpha}{\otimes} B)) \\
& =\sigma(P(A \otimes I, I \otimes B))=\sigma(\tilde{P}(A \otimes I, I \otimes B)) \\
& =\sigma\left(\sum c_{j k} A^{j} \underset{a}{\hat{\otimes}} B\right)=\sigma\left(\left(\sum c_{j k} A^{j} \underset{a}{\hat{\otimes}} B^{k}\right)^{\sim}\right) .
\end{aligned}
$$

Combined with Theorem 1.8 for faithful $\alpha$, we have

Corollary 3.3. Under the same condition of Theorem 3.2, suppose further it does not occur that one of the extended spectra $\sigma_{e}(A)$ and $\sigma_{e}(B)$ contains 0 while the other contains $\infty$. Then for $P \in \mathcal{P}(A, B)$

$$
\begin{aligned}
P(\sigma(A), \sigma(B)) & =P(\sigma(A \underset{a}{\hat{\otimes}} I), \sigma(I \underset{a}{\hat{\otimes}} B)) \\
& =\sigma(P(A \underset{a}{\hat{\otimes}} I, I \underset{a}{\hat{\otimes}} B))=\sigma(\widetilde{P}(A \underset{a}{\hat{\otimes}} I, I \underset{a}{\hat{\otimes}} B)) .
\end{aligned}
$$

Remark 3.4. The inclusion $P(\sigma(A), \sigma(B)) \subset \sigma\left(\sum c_{j k} A^{j} \hat{\otimes}_{a} B^{k}\right)$ in Theorem 3.2 may be proved also just in the same way as Theorem 3.6 in [10], if we note (1.4).

In Corollary 3.3, when $P(\sigma(A), \sigma(B)) \neq \mathbf{C}$ and $\lambda \notin P(\sigma(A), \sigma(B))$, the continuous inverse of the closure $\widetilde{P}\left(A \hat{\otimes}_{\alpha} I, I \hat{\otimes}_{\alpha} B\right)-\lambda I \hat{\otimes}_{\alpha} I$ may be given also by the integral

$$
(2 \pi i)^{-2} \int_{\partial U} \int_{\partial V}(P(\xi, \eta)-\lambda)^{-1} R(\xi ; A \underset{\alpha}{\hat{\otimes}} I) R(\eta ; \underset{\alpha}{\hat{\otimes}} B) d \xi d \eta
$$

where the open sets $U \supset \sigma(A)=\sigma\left(A \hat{\hat{\otimes}}_{\alpha} I\right)$ and $V \supset \sigma(B)=\sigma\left(I \hat{\otimes}_{\alpha} B\right)$ are chosen in the same way as in the proof of Theorem 2.1 . 
4. Polynomial operators of the first order. We remark first that the generalization of the results of the preceding sections to polynomials of $n$ variables is straightforward (cf. [10, Theorem 3.6]).

In this section we consider in particular polynomials of the first order. The corresponding operators were treated as separable operators by H. O. Cordes [4], B. Friedman [5], Ju. M. Berezanskĩ [2], L. and K. Maurin [16].

4.1. Spectral mapping theorem. $E$ being a Banach space, a densely defined closed linear operator $T: D[T] \subset E \rightarrow E$ is said to be of type $\left(\theta_{T}, M(\theta)\right)$, $0 \leq \theta_{T}<\pi$, if the resolvent set $\rho(T)$ contains the complement of the sector $S\left(\theta_{T}\right)=\left\{\zeta ;|\arg \zeta| \leq \theta_{T}\right\}$ and $\|\zeta R(\zeta ; T)\| \leq M(\theta), \theta=\arg \zeta$, outside $S\left(\theta_{T}^{\prime}\right)$ for each $\theta_{T}^{\prime}$ with $\theta_{T}<\theta_{T}^{\prime}<\pi$, where $M(\theta)$ is a constant depending only on $\theta=$ $\arg \zeta([11],[20])$.

The following theorems generalize the results of Ju. M. Berezanskii and L. and K. Maurin for selfadjoint operators in Hilbert spaces.

Theorem 4.1. Let $X_{j}, 1 \leq j \leq n$, be complex Banach spaces and a aniform reasonable norm on $X_{1} \otimes \cdots \otimes X_{n}$. For $j=1,2, \cdots, n$, let $A_{j}: D\left[A_{j}\right] \subset$ $X_{j} \rightarrow X_{j}$ be a densely defined closed linear operator of type $\left(\theta_{j}, M_{j}(\theta)\right)$ for which $0 \leq \theta_{j}<\pi$ and $0 \leq \theta_{j}+\theta_{k}<\pi$ for $j \neq k$ and $j, k=1,2, \cdots, n$. Then

$$
\begin{aligned}
\sum_{j=1}^{n} \sigma\left(A_{j}\right) & =\sigma\left(\sum_{j=1}^{n} I_{1} \otimes \cdots \otimes I_{j-1} \otimes A_{j} \otimes I_{j+1} \otimes \cdots \otimes I_{n}\right) \\
& =\sigma\left(\left(\sum_{j=1}^{n} I_{1} \otimes \cdots \otimes I_{j-1} \otimes A_{j} \otimes I_{j+1} \otimes \cdots \otimes I_{n}\right)^{\sim}\right),
\end{aligned}
$$

provided none of the $\sigma\left(A_{j}\right)$ are empty.

The spectra of $\sum_{j=1}^{n} I_{1} \otimes \cdots \otimes A_{j} \otimes \cdots \otimes I_{n}$ and its maximal extension $\left(\sum_{j=1}^{n} I_{1} \otimes \cdots \otimes A_{j} \otimes \cdots \otimes I_{n}\right)^{\sim}$ are empty if and only if at least one of the $\sigma\left(A_{j}\right)$ is empty.

Proof. Evidently the (vector) sum $\sum_{j=1}^{n} \sigma\left(A_{j}\right)$ is closed in $\mathbf{C}$ and does not cover the whole complex plane $\mathrm{C}$. Let $\lambda \notin \sum_{j=1}^{n} \sigma\left(A_{j}\right)$, so that $\delta=$ $\operatorname{dist}\left(\lambda, \sum_{j=1}^{n} \sigma\left(A_{j}\right)\right)>0$. If at least one of the $\sigma\left(A_{j}\right)$ is empty, $\lambda$ shall be an arbitrary complex number. We shall show for this $\lambda$ that we can find those open sets $U_{j}$ such as in the proof of Theorem 2.1.

Choose $\epsilon>0$ such that $\left(\theta_{j}+\epsilon\right)+\left(\theta_{k}+\epsilon\right)=\theta_{j}+\theta_{k}+2 \epsilon<\pi$ for $j \neq k$ and $j, k=1,2, \cdots, n$. Set $R=(|\lambda|+1) \operatorname{cosec}\left(\max _{j \neq k}\left(\theta_{j}+\theta_{k}+2 \epsilon\right)\right)$. Then for any $k$ the vector sum of the sectors

$$
S\left(\theta_{1}+\epsilon\right)+\cdots+S\left(\theta_{k-1}+\epsilon\right)+S\left(\theta_{k}+\epsilon\right)_{R}+S\left(\theta_{k+1}+\epsilon\right)+\cdots+S\left(\theta_{n}+\epsilon\right)
$$

is away from the origin at distance $\geq|\lambda|+1$, where $S\left(\theta_{k}+\epsilon\right)_{R}=S\left(\theta_{k}+\epsilon\right) \cap$ 
$\mathcal{C}_{K}(0 ; R) .\left|\sum_{j=1}^{n} \zeta_{j}-\lambda\right|$ is bounded away from zero when $\zeta=\left(\zeta_{1}, \cdots, \zeta_{n}\right)$ is in $\mathrm{Il}_{j=1}^{n} G_{j}$, where $G_{j}=\left\{\zeta\right.$; $\left.\operatorname{dist}\left(\zeta, \sigma\left(A_{j}\right)\right)<\delta / 2 n\right\}$ if $\sigma\left(A_{j}\right) \neq \emptyset$ and $G_{j}=$ $s\left(\theta_{j}+\epsilon\right)_{R}$ if $\sigma\left(A_{j}\right)=\varnothing$. For each $j, V_{j}$ shall be a bounded open set whose boundary consists of a finite number of rectifiable Jordan curves such that $\sigma\left(A_{j}\right)$ $\cap K(0 ; R) \subset V_{j} \subset G_{j}$. If $\sigma\left(A_{j}\right) \cap K(0 ; R)=\varnothing$, let $V_{j}=\varnothing$. As $U_{j}$, we choose the interior of the set $\left(V_{j} \cap K(0 ; R)\right) \cup S\left(\theta_{j}+\epsilon\right)_{R}$. Obviously, $\left(\sum_{j=1}^{n} \zeta_{j}-\lambda\right)\left(\sum_{j=1}^{n}\left|\zeta_{j}\right|\right)^{-1}$ is bounded away in absolute value from zero on $\Pi_{j=1}^{n} \bar{U}_{j}$ for sufficiently large $\sum_{j=1}^{n}\left|\zeta_{j}\right| \cdot \quad$ Q.E.D.

If $\alpha$ is faithful, we denote $I_{1} \otimes \ldots \otimes I_{j-1} \otimes A_{j} \otimes I_{j+1} \otimes \cdots \otimes I_{n}$ by $\mathbf{A}_{j}$ and its closure in $X_{1} \otimes \ldots \otimes X_{n}$ by $\tilde{\mathbf{A}}_{j}$ for $j=1,2, \cdots, n$.

The following theorem is a direct consequence of Theorem 3.2.

Theorem 4.2. Under the same condition as in Theorem 4.1, suppose further a is faitbful. Then

$$
\begin{aligned}
\sum_{j=1}^{n} \sigma\left(A_{j}\right) & =\sum_{j=1}^{n} \sigma\left(\tilde{\mathbf{A}}_{j}\right)=\sigma\left(\sum_{j=1}^{n} \mathbf{A}_{j}\right)=\sigma\left(\left(\sum_{j=1}^{n} \mathbf{A}_{j}\right) \sim\right) \\
& =\sigma\left(\sum_{j=1}^{n} \tilde{\mathbf{A}}_{j}\right)=\sigma\left(\left(\sum_{j=1}^{n} \tilde{\mathbf{A}}_{j}\right) \sim\right)
\end{aligned}
$$

provided none of the $\sigma\left(A_{j}\right)$ are empty. The spectra of $\sum_{j=1}^{n} \mathbf{A}_{j}, \sum_{j=1}^{n} \tilde{\mathbf{A}}_{j}$ and their closures are empty if and only if at least one of the $\sigma\left(A_{j}\right)$ is empty.

4.2. Fractional powers. If $A$ and $B$ satisfy the condition of Theorem 4.1 or 4.2 for $n=2$, we see by Theorem 3.1 that the closures of $A \otimes I+I \otimes B$ and of $A \hat{\otimes}_{\alpha} I+I \hat{\otimes}_{\alpha} \dot{B}$ coincide if $\alpha$ is a faithful uniform reasonable norm on $X \otimes Y$. It is an important question when this closure coincides with $A \hat{\otimes}_{a} I+I \hat{\otimes}_{a} B$ itself (cf. [3]).

Obviously they coincide if and only if $A \hat{\otimes}_{a} I+I \hat{\otimes}_{a} B$ is closed in $X \hat{\otimes}_{a} Y$. Note this is valid in virtue of the Banach-Schauder theorem if and only if the following inequality holds:

$$
\|A \underset{a}{\hat{\otimes}} I u\|_{a} \leq C_{A}\left[\|(A \underset{a}{\hat{\otimes}} I+I \underset{a}{\hat{\otimes}} B) u\|_{a}+\|u\|_{a}\right]
$$

or equivalently

$$
\|I \underset{\alpha}{\hat{\otimes}} B u\|_{\alpha} \leq C_{B}\left[\|(A \underset{\alpha}{\hat{\otimes}} I+I \underset{a}{\hat{\otimes}} B) u\|_{a}+\|u\|_{a}\right]
$$

for $u \in D\left[A \hat{\otimes}_{a} I\right] \cap D\left[I \hat{\otimes}_{\alpha} B\right]$, with constants $C_{A}$ and $C_{B}$.

We shall now derive a weaker inequality than (4.1) or (4.2), considering the fractional powers of operators.

If $A$ is of type $\left(\theta_{A}, M_{A}(\theta)\right)$, the fractional power $A^{\mu}, 0<\mu<1$, of $A$ is defined (cf. [11], [20]): 


$$
A^{\mu} x=\frac{\sin \pi \mu}{\pi} \int_{0}^{\infty} \zeta^{\mu-1}(\zeta I+A)^{-1} A x d \zeta, \quad x \in D[A]
$$

Deforming the integration contour, we can show easily that (4.3) equals

$$
A^{\mu} x=(2 \pi i)^{-1} \int_{\Gamma} \zeta^{\mu-1} R(\xi ; A) A x d \zeta, \quad x \in D[A],
$$

where the contour $\Gamma_{\overline{1}}=\left\{\zeta_{\text {; }}|\arg \zeta|=\theta_{T}^{\prime}\right\}, \theta_{T}<\theta_{T}^{\prime}<\pi$, runs from $\infty e^{i \theta_{T}^{\prime}}$ to 0 and from 0 to $\infty e^{-i \theta_{T}^{\prime}}$ and the branch of $\zeta^{\mu-1}$ is so taken that $\operatorname{Re} \zeta^{\mu-1}>0$ for $\operatorname{Re} \zeta>0$.

Lemma 4.3. Let a be a faitbful uniform reasonable norm on $X \otimes Y$. If $A: D[A] \subset X \rightarrow X$ is a densely defined closed linear operator of type $\left(\theta_{A}, M_{A}(\theta)\right)$ with $0 \leq \theta_{A}<\pi$, so is $A \hat{\otimes}_{a} I$. For $0 \leq \mu \leq 1$, we have $\left(A \hat{\otimes}_{a} I\right)^{\mu}=A^{\mu} \hat{\otimes}_{a} I$.

Proof. Note $\sigma\left(A \hat{\otimes}_{a} I\right)=\sigma(A)$ and $R\left(\zeta ; A \hat{\otimes}_{a} I\right)=R(\zeta ; A) \hat{\otimes}_{a} I$ for $\zeta \epsilon$ $\rho\left(A \hat{\otimes}_{\alpha} l\right)=\rho(A)$ [9]. Then the first assertion is evident. The second assertion for $\mu=0$ and $\mu=1$ is trivial. To prove it for $0<\mu<1$, we use the formula of the resolvent of the fractional power by T. Kato ([11], [20]). Then we have $\left[\lambda I \underset{\alpha}{\hat{\otimes}} I+(A \underset{\alpha}{\hat{\otimes}} I)^{\mu}\right]^{-1}=\left(\lambda I+A^{\mu}\right)^{-1} \underset{\alpha}{\hat{\otimes}} I=\left[\left(\lambda I+A^{\mu}\right) \underset{\alpha}{\underset{\otimes}{\otimes} I} I\right]^{-1}=\left[\lambda I \underset{\alpha}{\hat{\otimes}} I+A^{\mu} \underset{\alpha}{\hat{\otimes}} I\right]^{-1}$ whence $\left(A \hat{\otimes}_{a} I\right)^{\mu}=A^{\mu} \hat{\otimes}_{a} I$. Q.E.D.

Under the same condition as in Theorem 4.2 for $n=2$. we have

Theorem 4.4. Let $X$ and $Y$ be complex Banach spaces and a a faithful uniform reasonable norm on $X \otimes Y$. Let $A: D[A] \subset X \rightarrow X$ and $B: D[B] \subset Y \rightarrow Y$ be densely defined closed linear operators of type $\left(\theta_{A}, M_{A}(\theta)\right),\left(\theta_{B}, M_{B}(\theta)\right)$, respectively, with $0 \leq \theta_{A}+\theta_{B}<\pi$. Then if $0<\mu<1$, we have for $u \in D[A] \otimes D[B]$

$$
\left\|(A \underset{\alpha}{\hat{\otimes}} I)^{\mu} u\right\|_{\alpha}=\left\|A^{\mu} \underset{\alpha}{\hat{\otimes}} I u\right\|_{\alpha} \leq C_{\mu}\left[\|(A \otimes I+I \otimes B) u\|_{\alpha}+\|u\|_{\alpha}\right]
$$

and

$$
\left\|(I \underset{a}{\hat{z}} B)^{\mu} u\right\|_{a}=\left\|I \underset{a}{\hat{\otimes}} B^{\mu} u\right\|_{a} \leq C_{\mu}\left[\|(A \otimes I+I \otimes B) u\|_{a}+\|u\|_{a}\right]
$$

with a constant $C_{\mu}$ depending only on $\mu$.

Therefore for $\lambda \notin \sigma(A)+\sigma(B)$,

$$
\left[(A \otimes I+I \otimes B)^{2}-\lambda I \underset{a}{\hat{\otimes}} I\right]^{-1}=\left[(A \underset{a}{\hat{\otimes}} I+I \underset{\alpha}{\hat{\otimes}} B)^{2}-\lambda I \underset{a}{\hat{\otimes}} I\right]^{-1}
$$

is a one-to-one continuous linear operator of $X \hat{\otimes}_{a} Y$ onto $\mathbf{D} \equiv D\left[(A \otimes I+I \otimes B)^{2}\right]$ $C D\left[\left(A \hat{\otimes}_{a} I\right)^{\mu}\right] \cap D\left[\left(I \hat{\otimes}_{a} B\right)^{\nu}\right]$ for $0<\mu<1$ and $0<\nu<1$, where $\mathbf{D}$ is equipped with the topology induced by $D\left[\left(A \hat{\otimes}_{a} I\right)^{\mu}\right] \cap D\left[\left(I \hat{\otimes}_{a} B\right)^{\nu}\right]$, i.e. with the norm $\|u\|_{a}+\left\|\left(A \hat{\otimes}_{a} I\right)^{\mu} u\right\|_{a}+\left\|\left(I \hat{\otimes}_{a} B\right)^{\nu} u\right\|_{\alpha}$.

Proof. By Theorems 3.1 and 4.1 we have only to establish the inequalities 
(4.4) and (4.5). Since $-1 \notin \sigma(A)+\sigma(B)$, we see by Theorem 4.2 that the closure of $A \otimes I+I \otimes B+I \otimes I$ has a continuous inverse and maps onto $X \hat{\otimes}_{\alpha} Y$ :

Choose $\epsilon>0$ such that $\theta_{A}+\theta_{B}+2 \epsilon<\pi$. Let $v \in D[A] \otimes Y$. Then if we take (4.3) into account, for $0<\mu<1$ the integral

$$
(2 \pi i)^{-2} \int_{\Gamma_{A}} \int_{\Gamma_{B}} \frac{\xi^{\mu-1}}{\xi+\eta+1}[R(\xi ; A) A \otimes R(\eta ; B)] v d \xi d \eta
$$

where $\Gamma_{A}=\left\{\xi ;|\arg \xi|=\theta_{A}+\epsilon\right\}$ and $\Gamma_{B}=-1 / 2+\left\{\eta ;|\arg \eta|=\theta_{B}+\epsilon\right\}$ is absolutely convergent and defines a continuous linear operator of $D[A] \otimes Y \subset X \hat{\otimes}_{a} Y$ into $X \hat{\otimes}_{a} Y$. We denote its continuous extension to $X \hat{\otimes}_{\alpha} Y$ by $\mathbb{A}_{\mu}$.

Then similarly to the proof of Theorem 2.1 we have, for $u \in D\left[A^{2}\right] \otimes D\left[B^{2}\right]$ and $\eta_{0} \notin S\left(\theta_{\dot{B}}+\epsilon\right)-1 / 2$,

$$
\begin{aligned}
& \mathscr{Q}_{\mu}(A \otimes I+I \otimes B+I \otimes I)^{\sim} u \\
& =\mathscr{Q}_{\mu}(A \otimes I+I \otimes B+I \otimes I) u \\
& =(2 \pi i)^{-2} \int_{\Gamma_{A}} \int_{\Gamma_{B}} \frac{\xi^{\mu-1}}{\xi+\eta+1}[R(\xi ; A) A \otimes R(\eta ; B)] \\
& \cdot[A \otimes I+I \otimes B+I \otimes I] u d \xi d \eta \\
& =(2 \pi i)^{-2} \int_{\mathbf{r}_{A}} \int_{\Gamma_{B}} \frac{\xi^{\mu-1}}{(\xi+\eta+1)\left(\eta-\eta_{0}\right)}\left[R(\xi ; A) A \otimes R(\eta ; B)\left(B-\eta_{0} I\right)\right] \\
& \cdot[A \otimes I+I \otimes B+I \otimes I] u d \xi d \eta \\
& =(2 \pi i)^{-2} \int_{\Gamma_{A}} \int_{\Gamma_{B}} \xi^{\mu-1}\left(\eta-\eta_{0}\right)^{-1}\left[A R(\xi ; A) \otimes\left(B-\eta_{0} I\right) R(\eta ; B)\right] u d \xi d \eta \\
& +(2 \pi i)^{-2} \int_{\Gamma_{A}} \int_{\Gamma_{B}} \frac{\xi^{\mu-1}}{(\xi+\eta+1)\left(\eta-\eta_{0}\right)}\left[A R(\xi ; A) \otimes\left(B-\eta_{0} I\right) R(\eta ; B)\right] \\
& \cdot[(A-\xi I) \otimes I+I \otimes(B-\eta I)] u d \xi d \eta \\
& =\left(A^{\mu} \otimes I\right) u \text {, }
\end{aligned}
$$

for the second integral vanishes. Hence for $u \in D\left[A^{2}\right] \otimes D\left[B^{2}\right]$

$$
\begin{aligned}
\left\|\left(A^{\mu} \otimes I\right) u\right\|_{\alpha} & \leq\left\|\mathbb{C}_{\mu}\right\|\|(A \otimes I+I \otimes B+I \otimes I) u\|_{a} \\
& \leq C_{\mu}\left[\|(A \otimes I+I \otimes B) u\|_{\alpha}+\|u\|_{a}\right] .
\end{aligned}
$$

By Lemma 2.3, we see $D\left[A^{2}\right] \otimes D\left[B^{2}\right]$ is dense in $D[A] \otimes D[B]$ with respect to the graph norm of $A \otimes I+I \otimes B$, so that by the closedness of $A^{\mu} \hat{\otimes}_{a} I$ the above inequality is valid for $u \in D[A] \otimes D[B]$. This establishes (4.4) with Lemma 4.3. (4.5) will be shown just in the same way. Q.E.D.

4.3. m-accretive operators in Hilbert spaces. In the following, a particular case where the inequalities (4.1) and (4.2) hold will be considered. Here, $X$ and 
$Y$ are assumed to be Hilbert spaces and $\alpha_{0}$ the uniform crossnorm on $X \otimes Y$ such that $X \hat{\otimes}_{a_{0}} Y$ is a Hilbert space [18]. $\alpha_{0}$ is faithful. For the norm $\|\cdot\|_{a_{0}}$ we shall use the abbreviation $\|\cdot\|$.

Lemma 4.5. Let $A: D[A] \subset X \rightarrow X ; B: D[B] \subset Y \rightarrow Y$ be densely defined linear operators.

(1) If $A$ and $B$ are symmetric, so is $A \otimes B$ in $X \hat{\otimes}_{a_{0}} Y$.

(2) If $A$ is accretive, so is $A \otimes I$ in $X \hat{\otimes}_{a_{0}} Y$.

Proof. (1) Let $u \in D[A] \otimes D[B], u=\sum_{j=1}^{r} x_{j} \otimes y_{j}$, where both the sequences $\left\{x_{j}\right\}_{j=1}^{r} \subset D[A]$ and $\left\{y_{j}\right\}_{j=1}^{r} \subset D[B]$ are linearly independent. Then

$$
\begin{aligned}
((A \otimes B) u, u) & =\left(\sum_{j} A x_{j} \otimes B y_{j}, \sum_{j} x_{j} \otimes y_{j}\right)=\sum_{j, k}\left(A x_{j}, x_{k}\right)\left(B y_{j}, y_{k}\right) \\
& =\sum_{j, k}\left(x_{j}, A x_{k}\right)\left(y_{j}, B y_{k}\right)=\left(\sum_{j} x_{j} \otimes y_{j}, \sum_{j} A x_{j} \otimes B y_{j}\right) \\
& =(u,(A \otimes B) u) .
\end{aligned}
$$

(2) Let $u \in D[A] \otimes Y, u=\sum_{j=1}^{r} x_{j} \otimes y_{j}$, where both the sequences $\left\{x_{j}\right\}_{j=1}^{r} \subset$ $D[A]$ and $\left\{y_{j}\right\}_{j=1}^{r} \subset Y$ are linearly independent and further we may assume the latter is orthogonal. Then

$$
\operatorname{Re}((A \otimes I) u, u)=\operatorname{Re} \sum_{j}\left(A x_{j}, x_{j}\right)\left(y_{j}, y_{j}\right) \geq 0 . \quad \text { Q.E.D. }
$$

An m-accretive operator $A: D[A] \subset X \rightarrow X$ is by definition a closed accretive operator whose resolvent set $\rho(A)$ contains the left half-plane with $\left\|(A+\lambda I)^{-1}\right\|$ $\leq(\operatorname{Re} \lambda)^{-1}$ for $\operatorname{Re} \lambda>0$. It is necessarily densely defined. An m-sectorial operator $A: D[A] \subset X \rightarrow X$ with vertex 0 and semiangle $\theta_{A}, 0 \leq \theta_{A}<\pi / 2$, is by definition an $m$-accretive operator with

$$
|\operatorname{Im}(A x, x)| \leq \tan \theta_{A} \cdot \operatorname{Re}(A x, x), \text { for } x \in D[A]
$$

Then the real part $H_{A}=\operatorname{Re} A$ is nonnegative selfadjoint, and there exists a bounded selfadjoint operator $S_{A}$ such that $\left\|S_{A}\right\| \leq \tan \theta_{A}$ and $A=G_{A}\left(I+i S_{A}\right) G_{A}$ with $G_{A}=H_{A}^{1 / 2}$ (see [12]).

The following theorem may be considered as a generalization of the results by V. P. Mihaillov [17] for the boundary value problem of quasi-elliptic differential equations.

Theorem 4.6. Let $A: D[A] \subset X \rightarrow X$ and $B: D[B] \subset Y \rightarrow Y$ be m-accretive operators. Either 
(1) if one of $A$ and $B$ is selfadjoint, or

(2) if $A$ and $B$ are m-sectorial with semiangles $\theta_{A}, \theta_{B}$, respectively, sucb that $\tan \theta_{A} \cdot \tan \theta_{B} \leq 1$, then the closure of $A \otimes I+I \otimes B$ coincides with $A \hat{\otimes}_{a_{0}} I+I \hat{\otimes}_{a_{0}} B$ and

$$
\sigma(A)+\sigma(B)=\sigma\left(A \underset{a_{0}}{\hat{\otimes}} I\right)+\sigma\left(I \underset{a_{0}}{\hat{\otimes}} B\right)=\sigma\left(A \underset{a_{0}}{\hat{\otimes}} I+I \underset{a_{0}}{\hat{\otimes}} B\right) .
$$

In this case, if $\lambda \notin \sigma(A)+\sigma(B)$, for any $f \in X \hat{\otimes}_{a_{0}} Y$ there exists a unique $u \in D\left[A \hat{\otimes}_{a_{0}} I\right] \cap D\left[I \hat{\otimes}_{a_{0}} B\right]$ which satisfies $\left[A \hat{\otimes}_{a_{0}} I+I \hat{\otimes}_{a_{0}} B-\lambda I \hat{\otimes}_{a_{0}} I\right] u=f$. Moreover the following inequality bolds:

$$
\|u\|+\left\|A \underset{a_{0}}{\hat{\otimes}} I u\right\|+\left\|I \underset{a_{0}}{\hat{\otimes}} B u\right\| \leq C\|f\|,
$$

with a constant $C$ independent of $u$ and $f$.

Proof. In virtue of Theorem 4.2, we have only to establish

$$
\|(I \otimes B) u\| \leq C[\|(A \otimes I+I \otimes B) u\|+\|u\|],
$$

for $u \in D[A] \otimes D[B]$. To do so it suffices to show that $\operatorname{Re}((A \otimes I) u,(I \otimes B) u)$ $\geq 0$ for $u \in D[A] \otimes D[B]$. For then it will follow that

$$
((A \otimes I) u,(I \otimes B) u)+((I \otimes B) u,(I \otimes B) u)=(f,(I \otimes B) u)
$$

where $u \in D[A] \otimes D[B]$ and $f=(A \otimes I+I \otimes B) u$, whence we shall be able to derive (4.2)' easily.

(1) Without loss of generality we may assume that $A$ is $m$-accretive and $B$ is nonnegative, selfadjoint.

For $u \in D[A] \otimes D[B], u=\sum_{j=1}^{r} x_{j} \otimes y_{j}$, where $\left\{x_{j}\right\}_{j=1}^{r} \subset D[A]$ and $\left\{y_{j}\right\}_{j=1}^{r}$ $C D[B]$, we have by Lemma 4.5

$$
\begin{aligned}
\operatorname{Re}((A \otimes I) u,(I \otimes B) u)=\operatorname{Re}\left(\sum_{j} A x_{j} \otimes y_{j}, \sum_{j} x_{j} \otimes B y_{j}\right) \\
\quad=\operatorname{Re} \sum_{j, k}\left(A x_{j}, x_{k}\right)\left(y_{j}, B y_{k}\right)=\operatorname{Re} \sum_{j, k}\left(A x_{j}, x_{k}\right)\left(B^{1 / 2} y_{j}, B^{1 / 2} y_{k}\right) \\
\quad=\operatorname{Re}\left((A \otimes I)\left(I \otimes B^{1 / 2}\right) u,\left(I \otimes B^{1 / 2}\right) u\right) \\
\quad \geq 0 .
\end{aligned}
$$

(2) There exist bounded selfadjoint operators $S_{A}, S_{B}$ such that $\left\|S_{A}\right\| \leq$ $\tan \theta_{A},\left\|S_{B}\right\| \leq \tan \theta_{B}$ and $A=G_{A}\left(I+i S_{A}\right) G_{A}, G_{A}=H_{A}^{1 / 2} ; \quad B=G_{B}\left(I+i S_{B}\right) G_{B^{\prime}}$, $G_{B}=H_{B}^{1 / 2}$. Let $u \in D[A] \otimes D[B]$ as in the proof (1). Since $G_{A}$ and $G_{B}$ are selfadjoint, and since $S_{A}$ and $S_{B}$ are bounded, selfadjoint, we have 


$$
\begin{aligned}
((A \otimes & I) u,(I \otimes B) u) \\
= & \sum_{j, k}\left(A x_{j}, x_{k}\right)\left(y_{j}, B y_{k}\right) \\
= & \sum_{j, k}\left(\left(I+i S_{A}\right) G_{A} x_{j}, G_{A} x_{k}\right)\left(G_{B} y_{j},\left(I+i S_{B}\right) G_{B} y_{k}\right) \\
= & \sum_{j, k}\left[\left(G_{A} x_{j}, G_{A} x_{k}\right)\left(G_{B} y_{j}, G_{B} y_{k}\right)+\left(S_{A} G_{A} x_{j}, G_{A} x_{k}\right)\left(G_{B} y_{j}, S_{B} G_{B} y_{k}\right)\right] \\
& +i \sum_{j, k}\left[\left(S_{A} G_{A} x_{j}, G_{A} x_{k}\right)\left(G_{B} y_{j}, G_{B} y_{k}\right)-\left(G_{A} x_{j}, G_{A} x_{k}\right)\left(G_{B} y_{j}, S_{B} G_{B} y_{k}\right)\right] \\
= & \sum_{j, k}\left[\left(G_{A} x_{j}, G_{A} x_{k}\right)\left(G_{B} y_{j}, G_{B} y_{k}\right)+\left(S_{A} G_{A} x_{j}, G_{A} x_{k}\right)\left(S_{B} G_{B} y_{j}, G_{B} y_{k}\right)\right] \\
& +i \sum_{j, k}\left[\left(S_{A} G_{A} x_{j}, G_{A} x_{k}\right)\left(G_{B} y_{j}, G_{B} y_{k}\right)-\left(G_{A} x_{j}, G_{A} x_{k}\right)\left(S_{B} G_{B} y_{j}, G_{B} y_{k}\right)\right] \\
= & {\left[\left(\left(G_{A} \otimes G_{B}\right) u,\left(G_{A} \otimes G_{B}\right) u\right)+\left(\left(S_{A} \otimes S_{B}\right)\left(G_{A} \otimes G_{B}\right) u,\left(G_{A} \otimes G_{B}\right) u\right)\right] } \\
& +i\left[\left(\left(S_{A} \otimes I\right)\left(G_{A} \otimes G_{B}\right) u,\left(G_{A} \otimes G_{B}\right) u\right)\right. \\
& \left.\quad-\left(\left(I \otimes S_{B}\right)\left(G_{A} \otimes G_{B}\right) u,\left(G_{A} \otimes G_{B}\right) u\right)\right] .
\end{aligned}
$$

By Lemma 4.5 (1), both the expressions in the brackets $[\cdots]$ of the above last formula are real. Thus it follows by the uniformness of $\alpha_{0}$ that

$$
\begin{aligned}
\operatorname{Re} & ((A \otimes I) u,(I \otimes B) u j \\
& =\left(\left(G_{A} \otimes G_{B}\right) u,\left(G_{A} \otimes G_{B}\right) u\right)+\left(\left(S_{A} \otimes S_{B}\right)\left(G_{A} \otimes G_{B}\right) u,\left(G_{A} \otimes G_{B}\right) u\right) \\
& \geq\left(1-\left\|S_{A}\right\|\left\|S_{B}\right\|\right)\left(\left(G_{A} \otimes G_{B}\right) u,\left(G_{A} \otimes G_{B}\right) u\right) \\
& \geq\left(1-\tan \theta_{A} \cdot \tan \theta_{B}\right)\left(\left(G_{A} \otimes G_{B}\right) u,\left(G_{A} \otimes G_{B}\right) u\right) \geq 0 . \quad \text { Q.E.D. }
\end{aligned}
$$

$F$ inally, we note that our results may also be applied to the spectral theory of many-body Schrödinger operators (cf. [1], [1a]).

\section{REFERENCES}

1. E. Balslev and J. M. Combes, Spectral properties of many-body Schrödinger operators with dilatation-analytic interactions, Comm. Math. Phys. 22 (1971), 280-294.

1a. E. Balslev, Spectral the ory of Schrödinger operators of many-body systems with permutation and rotation symmetries, Ann. Physics (to appear).

2. Ju. M. Berezanskii, Expansion in eigenfunctions of selfadjoint operators, "Naukova Dumka", Kiev, 1965; English transl., Transl. Math. Monographs, vol. 17, Amer. Math. Soc., Providence, R. I., 1968. MR 36 \#5768; \#5769.

3. R. Carroll, Problems in linked operators. I, II, Math. Ann. 151 (1963), 272-282; ibid. 160 (1965), 233-256. MR 29 \#3902; 36 \#6981.

4. H. O. Cordes, Über die Spektralzerlegung von hypermaximalen Operatoren, die durch Separation der Variablen zerfallen. I, II, Math. Ann. 128 (1954), 257-289; 373-411. MR 16, 597.

5. B. Friedman, An abstract formulation of the method of separation of variables, Proc. Conference Differential Equations, University of Maryland, College Park, Md., 1956, pp. 209-226. MR 18, 584.

6. J. Gil de Lamadrid, Uniform cross norms and tensor products of Banach algebras, Duke Math. J. 32 (1965), 359-369. MR 32 \#8125. 
7. A. Grothendieck, Produits tensoriels topologiques et espaces nucléaires, Mem. Amer. Math. Soc. No. 16 (1955). MR 17, 763.

8. E. Hille and R. S. Phillips, Functional analysis and semi-groups, rev. ed., Amer. Math. Soc. Colloq. Publ., vol. 31, Amer. Math. Soc., Providence, R.I., 1957. MR 19, 664.

9. T. Ichinose, On the spectra of tensor products of linear operators in Banach spaces, J. Reine Angew. Math. 244 (1970), 119-153.

10. - Operational calculus for tensor product of linear operators in Banach spaces, Scripta Math. (to appear).

11. T. Kato, Note on fractional powers of linear operators, Proc. Japan Acad. 36 (1960), 94-96. MR $22 \# 12400$.

12. - , Perturbation theory for linear operators, Die Grundlehren der math. Wissenschaften, Band 132, Springer-Verlag, New York, 1966. MR 34 \#3324.

13. G. Köthe, Topologische lineare Räume.I, Zweite verbesserte Auflage, Die Grundlehren der math. Wis senschaften, Band 107, Springer-Verlag, New York, 1966. MR $33 \# 3069$.

14. - General linear trans formations of locally convex spaces, Math. Ann. 159 (1965), 309-328. MR 33 \#573.

15. C. C. MacDuffee, The theory of matrices, Springer, Berlin, 1933.

16. L. Maurin and K. Maurin, Spektraltheorie separierbarer Operatoren, Studia Math. 23 (1963), 1-29. MR 27 \#5123.

17. V. P. Mihaulov, The first boundary-value problem for a class of hypo-elliptic equations, Mat. Sb. 63 (105) (1964), 238-264. (Russian) MR 29 \#369.

18. R. Schatten, A theory of cross-spaces, Ann. of Math. Studies, no. 26, Princeton Univ. Press, Princeton, N. J., 1950. MR 12, 186。

19. M. Schechter, On the spectra of operators on tensor products, J. Functional Analysis 4 (1969), 95-99. MR 41 \#2431.

20. K. Yosida, Functional analysis, Die Grundlehren der math. Wissenschaften, Band 123, Academic Press, New York; Springer-Verlag, Berlin, 1965. MR 31 \#5054.

DEPARTMENT OF MATHEMATICS, AICHI UNIVERSITY OF EDUCATION, IGAYA-CHO, KARIYA-SHI, JAPAN 448 